\title{
Purinergic Signaling Controls Spontaneous Activity in the Auditory System throughout Early Development
}

\author{
Travis A. Babola, ${ }^{1 \dagger}$ Sally Li, ${ }^{1 \dagger}$ Zhirong Wang, ${ }^{2 \dagger}$ Calvin J. Kersbergen, ${ }^{1}$ Ana Belén Elgoyhen, ${ }^{3}$ Thomas M. Coate, ${ }^{2}$ \\ and Dwight E. Bergles ${ }^{1,4,5}$ \\ ${ }^{1}$ Solomon H. Snyder Department of Neuroscience, Johns Hopkins University, Baltimore, Maryland 21205, ${ }^{2}$ Department of Biology, Georgetown \\ University, Washington, DC 20007, ${ }^{3}$ Instituto de Investigaciones en Ingeniería Genética y Biología Molecular, Consejo Nacional de Investigaciones \\ Científicas y Técnicas, Buenos Aires, 1428, Argentina, ${ }^{4}$ Department of Otolaryngology Head and Neck Surgery, Johns Hopkins University, \\ Baltimore, Maryland 21287, and ${ }^{5}$ Johns Hopkins University Kavli Neuroscience Discovery Institute, Baltimore, Maryland 21205
}

\begin{abstract}
Spontaneous bursts of electrical activity in the developing auditory system arise within the cochlea before hearing onset and propagate through future sound-processing circuits of the brain to promote maturation of auditory neurons. Studies in isolated cochleae revealed that this intrinsically generated activity is initiated by ATP release from inner supporting cells (ISCs), resulting in activation of purinergic autoreceptors, $\mathrm{K}^{+}$efflux, and subsequent depolarization of inner hair cells. However, it is unknown when this activity emerges or whether different mechanisms induce activity during distinct stages of development. Here we show that spontaneous electrical activity in mouse cochlea from both sexes emerges within ISCs during the late embryonic period, preceding the onset of spontaneous correlated activity in inner hair cells and spiral ganglion neurons, which begins at birth and follows a base to apex developmental gradient. At all developmental ages, pharmacological inhibition of P2Y1 purinergic receptors dramatically reduced spontaneous activity in these three cell types. Moreover, in vivo imaging within the inferior colliculus revealed that auditory neurons within future isofrequency zones exhibit coordinated neural activity at birth. The frequency of these discrete bursts increased progressively during the postnatal prehearing period yet remained dependent on P2RY1. Analysis of mice with disrupted cholinergic signaling in the cochlea indicate that this efferent input modulates, rather than initiates, spontaneous activity before hearing onset. Thus, the auditory system uses a consistent mechanism involving ATP release from ISCs and activation of P2RY1 autoreceptors to elicit coordinated excitation of neurons that will process similar frequencies of sound.
\end{abstract}

Key words: a9; cochlea; inferior colliculus; P2RY1; potassium buffering; purinergic

Significance Statement

In developing sensory systems, groups of neurons that will process information from similar sensory space exhibit highly correlated electrical activity that is critical for proper maturation and circuit refinement. Defining the period when this activity is present, the mechanisms responsible and the features of this activity are crucial for understanding how spontaneous activity influences circuit development. We show that, from birth to hearing onset, the auditory system relies on a consistent mechanism to elicit correlate firing of neurons that will process similar frequencies of sound. Targeted disruption of this activity will increase our understanding of how these early circuits mature and may provide insight into processes responsible for developmental disorders of the auditory system.

Received Aug. 11, 2020; revised Oct. 6, 2020; accepted Nov. 25, 2020.

Author contributions: T.A.B. and D.E.B. designed research; T.A.B., S.L., Z.W., and C.J.K. performed research; T.A.B., S.L., Z.W., C.J.K., and T.M.C. analyzed data; T.A.B. wrote the first draft of the paper; T.A.B., S.L., C.J.K., A.B.E., T.M.C., and D.E.B. edited the paper; T.A.B. wrote the paper; A.B.E. contributed unpublished reagents/ analytic tools.

${ }^{\dagger}$ T.A.B., S. L., and Z.W. contributed equally to this work.

T.A.B. was supported by National Institutes of Health National Research Service Award F31DC016497. This work was supported by National Institutes of Health Grants DC008860 and NS050274 to D.E.B. and DC001508 to A.B.E., Mathers Foundation Grant MF-1804-00065, and Johns Hopkins University Rubenstein Fund for Hearing Research. We thank Dr. Michele Pucak and Naiqing Ye for technical assistance; Terry Shelley for machining expertise; and members of the D.E.B. laboratory for discussions and comments on the manuscript.

The authors declare no competing financial interests.

Correspondence should be addressed to Dwight E. Bergles at dbergles@jhmi.edu.

https://doi.org/10.1523/JNEUROSCI.2178-20.2020

Copyright $\odot 2021$ the authors

\section{Introduction}

In the developing CNS, spontaneous bursts of electrical activity promote maturation of newly formed neural circuits by promoting cell specification, survival, and refinement (Blankenship and Feller, 2010). These periodic bouts of electrical activity are prominent in developing sensory systems, where they arise through sensory-independent mechanisms. In the visual system, intrinsically generated bursts of electrical activity, termed retinal waves, sweep across the retina (Feller et al., 1996) and, when disrupted, lead to refinement deficits in higher visual centers (Rossi et al., 2001; Xu et al., 2011; Zhang et al., 2011). The mechanisms responsible for generating this activity are dynamic and progress 
through distinct stages before eye opening, with early waves mediated by gap-junction coupling and later by acetylcholine and glutamate release from starburst amacrine and bipolar cells, respectively (Firth et al., 2005; Blankenship and Feller, 2010). Across these stages, neural activity changes dramatically, progressing from individual propagating waves to complex wavelets that can be modulated by external light (Tiriac et al., 2018; Gribizis et al., 2019). These results demonstrate that the visual system uses an intricate process to shift activity patterns according to developmental stage, which may be optimized to achieve distinct aspects of circuit maturation. Although the visual system provides a template to understand developmental changes in sensory pathways, it is not known whether other sensory systems shift the mechanisms that induce spontaneous activity to create distinct patterns of neuronal firing at different developmental stages, limiting our understanding of how these circuits use this activity to induce maturation.

In the developing auditory system, peripheral and central neurons exhibit periodic bursts of action potentials that originate within the cochlea (Lippe, 1994; Tritsch et al., 2007, 2010). Before hearing onset, a group of glial-like inner supporting cells (ISCs) located adjacent to inner hair cells (IHCs) spontaneously release ATP, activating a metabotropic purinergic cascade that ultimately results in release of $\mathrm{K}^{+}$, IHC depolarization and subsequent burst firing of spiral ganglion neurons (SGNs) and central auditory neurons (Sonntag et al., 2009; Babola et al., 2018). Recent mechanistic studies revealed that activation of purinergic $\mathrm{P} 2 \mathrm{Y} 1$ receptors and downstream gating of $\mathrm{Ca}^{2+}$-activated chloride channels (TMEM16A) are required, and that correlated activity in central auditory circuits is sensitive to P2RY1 inhibition in vivo after the first postnatal week (H. C. Wang et al., 2015; Babola et al., 2020). Efferent inhibition of IHCs through activation of $\alpha 9$ subunit-containing nAChRs has also been implicated in both initiating (Johnson et al., 2012) and modulating (Clause et al., 2014) spontaneous activity during this period. Although SGNs can fire action potentials as early as E14.5 (Marrs and Spirou, 2012), it is not known when burst firing begins within the cochlea or what specific mechanisms initiate this activity at each developmental stage. Understanding these processes may help define the discrete steps required for maturation of precise auditory circuits and enhance our understanding of developmental auditory disorders.

Here, we examined the mechanisms responsible for initiating spontaneous activity in embryonic and postnatal mouse cochleae before hearing onset. Our results indicate that ISC electrical activity requires release of ATP and activation of P2Y1 autoreceptors at all developmental stages. Consistent with the critical role of ISC activation in triggering periodic excitation of IHCs and SGNs, acute pharmacological inhibition of P2RY1 disrupted correlated activation of IHCs and SGNs during this period. In vivo imaging of auditory midbrain neurons in neonatal awake mice revealed that neurons within future isofrequency zones exhibit correlated activity at birth, providing a 2 week period of highly stereotyped activity with which to influence circuit maturation. The frequency of these events increased progressively over the first 2 postnatal weeks but remained dependent on P2RY1. Together, these studies suggest that, in contrast to the developing visual system, the auditory system uses a persistent mechanism involving ISC ATP release and activation of purinergic autoreceptors to elicit periodic bursts of activity in discrete groups of sensory neurons that will process similar frequencies of sound.

\section{Materials and Methods}

Both male and female mice between embryonic day 14 (E14) and postnatal day 16 (P16) were used for all experiments and randomly allocated to experimental groups. All animals were healthy and were only used for experiments detailed in this study. Transgenic breeders were crossed to female FVB/NJ (Friend Virus B NIH Jackson; demonstrated low hearing thresholds at 28 weeks) mice to improve litter sizes and pup survival (Zheng et al., 1999). For these studies, all mouse lines were maintained on mixed backgrounds, except for Snap25-T2A-GCaMP6s mice used in Figures 7 and 8, which were maintained on a C57BL/6 background. Mice were housed on a $12 \mathrm{~h}$ light/dark cycle and were provided food ad libitum. This study was performed in accordance with recommendations provided in the Guide for the care and use of laboratory animals of the National Institutes of Health. All experiments and procedures were approved by the Johns Hopkins Institutional Care and Use Committee (protocol \#M018M330) and the Georgetown University Institutional Animal Care and Use Committee (protocol \#1147). Surgery was performed under isoflurane anesthesia, and extensive effort was made to minimize animal suffering.

Isolation of embryonic cochlear tissue. For imaging embryonic and neonatal SGNs, we used a cochlear dissection and culture protocol similar to that described previously (Driver and Kelley, 2010). Briefly, timedpregnant females were killed by $\mathrm{CO}_{2}$ at gestation days E14 to P0. Embryos were removed from the uterus immediately and kept in $1 \times$ Hanks' buffered saline solution/HEPES on ice. For calcium imaging, Snap25-T2A-GCaMP6s-positive pups were identified by their global epifluorescence signal. To isolate each cochlea for imaging, the cochlear capsule and stria were removed, and each cochlea was separated into api$\mathrm{cal}$ and basal sections. Cochleae were then transferred onto polycarbonate membrane filters (Sterlitech, PCT0213100) in a $14 \mathrm{~mm}$ bottom well dish with \#0 cover glass (In Vitro Scientific, D29-14-0-N) filled with $250 \mu \mathrm{l}$ media containing L-15 media (Invitrogen, 21083027), 10\% FBS, $0.2 \% \mathrm{~N}_{2}, 0.001 \%$ ciprofloxacin, and $0.1 \mathrm{~mm}$ Trolox. Cochlear pieces were flattened by surface tension and incubated at $37^{\circ} \mathrm{C}$ with $95 \% \mathrm{O}_{2} / 5 \% \mathrm{CO}_{2}$ for a minimum of 2 and maximum of $6 \mathrm{~h}$ before imaging. For electrophysiology experiments, dissections of cochleae were performed acutely in bicarbonate-buffered ACSF. Cochleae were placed immediately in the recording chamber and allowed to acclimate for $10 \mathrm{~min}$ in solutions at near physiological temperature $\left(32^{\circ} \mathrm{C}-34^{\circ} \mathrm{C}\right.$; see below).

Electrophysiology. For ISC recordings, apical and basal segments of the cochlea were acutely isolated from mouse pups and used within $2 \mathrm{~h}$ of the dissection. Cochleae were moved into a recording chamber and continuously superfused with bicarbonate-buffered ACSF $(1.5-2 \mathrm{ml} /$ min) consisting of the following (in $\mathrm{mM}$ ): $119 \mathrm{NaCl}, 2.5 \mathrm{KCl}, 1.3 \mathrm{MgCl}_{2}$, $1.3 \mathrm{CaCl}_{2}, 1 \mathrm{NaH}_{2} \mathrm{PO}_{4}, 26.2 \mathrm{NaHCO}_{3}, 11 \mathrm{D}$-glucose, and saturated with $95 \% \mathrm{O}_{2} / 5 \% \mathrm{CO}_{2}$ to maintain a $\mathrm{pH}$ of 7.4 . Near physiological temperature $\left(32^{\circ} \mathrm{C}-34^{\circ} \mathrm{C}\right)$ solutions were superfused using a feedback-controlled inline heater (Warner Instruments). Whole-cell recordings of ISCs were made under visual control using differential interference contrast (DIC) microscopy. Electrodes had tip resistances between 3.5 and $4.5 \mathrm{M} \Omega$ when filled with internal solution consisting of the following (in $\mathrm{mM}$ ): $134 \mathrm{KCH}_{3} \mathrm{SO}_{3}$, 20 HEPES, 10 EGTA, $1 \mathrm{MgCl}_{2}, 0.2 \mathrm{Na}-\mathrm{GTP}, \mathrm{pH}$ 7.3. Spontaneous currents were recorded with ISCs held at $-80 \mathrm{mV}$ and recorded for at least 5 min with pClamp10 software using a Multiclamp 700B amplifier, low pass filtered at $1 \mathrm{kHz}$, and digitized at $5 \mathrm{kHz}$ with a Digidata $1322 \mathrm{~A}$ analog-to-digital converter (Molecular Devices). Errors because of voltage drop across the series resistance and the liquid junction potential were left uncompensated for recordings of spontaneous activity.

For quantification of spontaneous events, traces were imported into MATLAB (Mathworks) and baseline-corrected using the msbackadj function ( $30 \mathrm{~s}$ window). Events were defined as peaks in the signal that exceed $20 \mathrm{pA}$ using the findpeaks function (minPeakProminence $=20$ ). Amplitude is represented as the mean amplitude and integral as the average charge transfer per second $(\mathrm{pA} / \mathrm{s})$. Input resistances were calculated by taking the change in voltage to a small negative current injection and dividing it by the amplitude of the current injection $(-100 \mathrm{pA})$.

For experiments with MRS2500 application, a 5 min baseline was collected before beginning flow of MRS2500 (1 $\mu \mathrm{M})$. After a 3 min washin period, the following $5 \mathrm{~min}$ period was used for MRS2500 analysis. 
Immunohistochemistry. Mice were deeply anesthetized with isoflurane and perfused with freshly prepared PFA (4\%) in $0.1 \mathrm{M} \mathrm{PB}$. Cochleae were postfixed overnight at room temperature and stored at $4^{\circ} \mathrm{C}$ in $\mathrm{PBS}$ until processing. For immunohistochemistry, P0-P11 cochleae were removed from the temporal bone and washed $3 \times 5$ min with PBS. Cochleae were incubated overnight with primary antibodies against $\beta$-gal (rabbit anti- $\beta$-galactosidase; 1:4000, Sanes laboratory) and calbindin (goat anti-calbindin; 1:500, Santa Cruz Biotechnology) for detection of $\beta$-galactosidase and visualization of hair cells. Cochleae were then rinsed 3 times with PBS and incubated for $2 \mathrm{~h}$ at room temperature with secondary antibodies raised in donkey (Alexa-488 and Alexa-546; 1:2000, Invitrogen). Slides were washed 3 times in PBS (second with PBS + 1:10,000 DAPI), allowed to dry, and sealed using Aqua Polymount (Polysciences). Images were captured using a laser scanning confocal microscope (LSM 880, Carl Zeiss).

Transmitted light imaging. Cochlear segments were imaged with an Olympus $40 \times$ water immersion objective (LUMPlanFl/IR) and recorded using MATLAB and a USB capture card (EZ Cap). Movies were generated by subtracting frames at time $t_{n}$ and $t_{n+5} s$ using MATLAB. To quantify transmittance changes, a threshold of 3 SDs above the mean was applied to each pixel value over time. To calculate the frequency of these events, the whole field was taken as an ROI and peaks detected using MATLAB (findpeaks function).

For experiments with MRS2500 application, a 10 min baseline was collected before beginning flow of MRS2500 $(1 \mu \mathrm{M})$. After a 5 min washin period, the following $10 \mathrm{~min}$ period was used for MRS2500 analysis. An additional washout period of $25 \mathrm{~min}$ was captured, with the final 10 min quantified as washout.

Cochlear explant culture. For imaging of ISCs and IHCs, cochleae were dissected from postnatal day 0 Pax2-Cre;R26-lsl-GCaMP3 mice in ice-cold, sterile-filtered HEPES-buffered ACSF consisting of the following (in mM): $130 \mathrm{NaCl}, 2.5 \mathrm{KCl}, 10$ HEPES, $1 \mathrm{NaH}_{2} \mathrm{PO}_{4}, 1.3 \mathrm{MgCl}_{2}, 2.5$ $\mathrm{CaCl}_{2}$, and $11 \mathrm{D}$-glucose. Explants were mounted onto Cell-Tak (Corning)-treated coverslips and incubated at $37^{\circ} \mathrm{C}$ for 2-6 h in DMEM (F-12/DMEM; Invitrogen) supplemented with $1 \% \mathrm{FBS}$ and $10 \mathrm{U} / \mathrm{ml}$ penicillin (Sigma Millipore) before imaging.

Confocal imaging of explants. For imaging of ISCs and IHCs, cochleae were moved into a recording chamber and continuously superfused with bicarbonate-buffered ACSF (1.5-2 $\mathrm{ml} / \mathrm{min})$ consisting of the following (in mM): $115 \mathrm{NaCl}, 6 \mathrm{KCl}, 1.3 \mathrm{MgCl}_{2}, 1.3 \mathrm{CaCl}_{2}, 1 \mathrm{NaH}_{2} \mathrm{PO}_{4}, 26.2$ $\mathrm{NaHCO}_{3}, 11 \mathrm{D}$-glucose, and saturated with $95 \% \mathrm{O}_{2} / 5 \% \mathrm{CO}_{2}$ to maintain a $\mathrm{pH}$ of 7.4. Images were captured at 2 frames per second using a laser scanning confocal microscope (LSM 710, Carl Zeiss) through a $20 \times$ objective (Plan APOCHROMAT $20 \times / 1.0 \mathrm{NA}$ ) at $512 \times 512$ pixels $(354 \times 354 \mu \mathrm{m} ; 16$ bit depth) resolution. Sections were illuminated with a $488 \mathrm{~nm}$ laser (maximum $25 \mathrm{~mW}$ power). MRS2500 (1 $\mu \mathrm{M}$, Tocris Bioscience) was applied by addition to superfused ACSF.

For imaging of SGNs, cultured cochlear pieces were removed from the incubator and placed with SGNs/IHCs facing down. Cochlear pieces were stabilized with a platinum harp with nylon strings. The bottom of the well was filled with $250 \mu \mathrm{l}$ static bath of ACSF consisting of the following (in mM): $145 \mathrm{NaCl}, 5 \mathrm{KCl}, 1 \mathrm{CaCl}_{2}, 1 \mathrm{MgCl}_{2}, 1 \mathrm{NaH}_{2} \mathrm{PO}_{4}, 5$ HEPES, and $5 \mathrm{D}$-glucose with a $\mathrm{pH}$ of 7.4. Imaging was performed at room temperature $\left(22^{\circ} \mathrm{C}-24^{\circ} \mathrm{C}\right)$. Images were captured at 1 frame per second using a laser scanning confocal microscope (LSM 880, Carl Zeiss) through a $20 \times$ objective (Plan-Apochromat $20 \times / 0.8 \mathrm{M} 27$ ) at $800 \times 800$ pixels $(708 \mu \mathrm{m} \times 708 \mu \mathrm{m}$; 16 bit depth, $1.32 \mu$ s dwell time) resolution. Tissues were illuminated with a $488 \mathrm{~nm}$ Argon laser with emission ranging from 500 to $540 \mathrm{~nm}$ and a GaAsp detector. Baseline imaging sessions consisted of 5 consecutive minutes of recording. Image acquisition was stopped, and CNQX (50 $\mu \mathrm{m}$; Sigma Millipore, C239) and CPP (100 $\mu \mathrm{M}$; Abcam, ab120159) or MRS2500 (1 $\mu \mathrm{M}$; Tocris Bioscience, 2154) were added directly to the bath and allowed to equilibrate for $5 \mathrm{~min}$ before capturing an additional $5 \mathrm{~min}$ used for analysis of pharmacological block.

Analysis of in vitro $\mathrm{Ca}^{2+}$ transients. For analysis of IHC and ISC activity, image stacks were imported into MATLAB where an ROI was drawn around the ISC and IHCs. For ISCs, a 10 pixel $\times 10$ pixel grid was imposed across the entire image, and only squares within the drawn
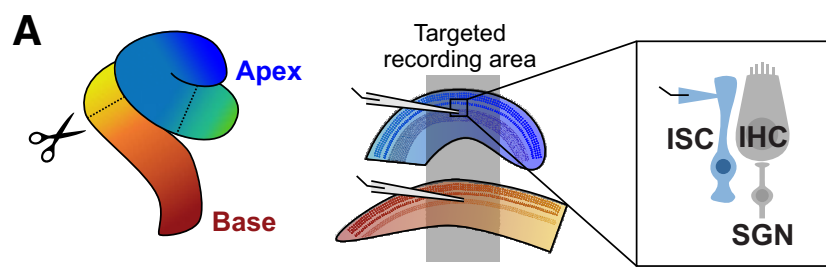

B
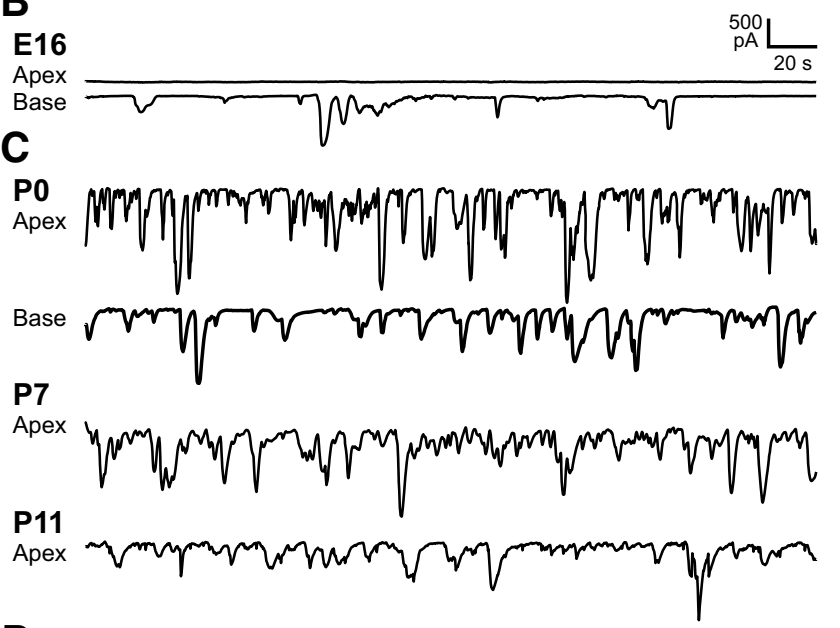

D
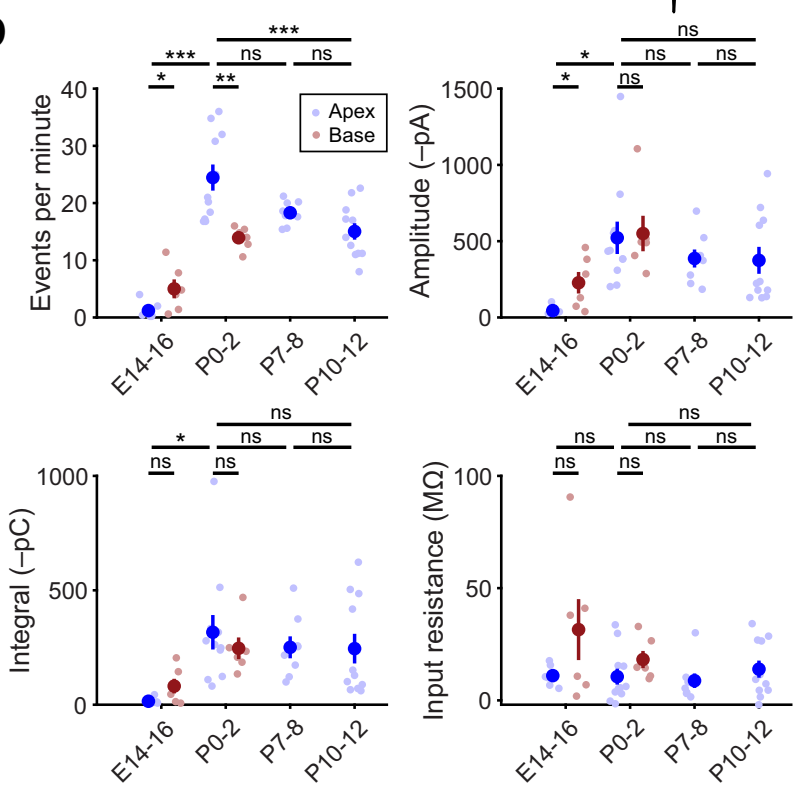

Figure 1. Prenatal onset of spontaneous activity in cochlear ISCS. A, Diagram of cochlea with approximate locations of cuts used for targeted apical and basal recordings of ISCS. Inset, Schematic of whole-cell recording configuration from ISCs. Recordings were made near physiological temperatures $\left(32^{\circ} \mathrm{C}-34^{\circ} \mathrm{C}\right)$. B , Exemplar voltage-clamp recordings of E16 apical and basal ISCS from the same cochlea. $\boldsymbol{C}$, Spontaneous inward currents in ISCS at different postnatal ages. D, Quantification of ISC spontaneous current frequency, amplitude, integral (charge transfer), and input resistance. Data are mean \pm SEM. $n=12$ E14-E16 recordings, 6 cochleae ( 6 apex and 6 paired base) from 6 mice; $n=11$ apical P0-2 recordings, 11 cochleae from 6 mice; $n=6$ basal P0-2 recordings, 6 cochleae from 6 mice; $n=8$ P7-8 recordings, 8 cochleae from 8 mice; and $n=11$ P10-12 recordings, 11 cochleae from 8 mice. Comparisons between E16 apex and base: $* p<0.05$; Mann-Whitney $U$ with Benjamini-Hochberg adjustment. For comparisons between ages, E14-E16 base and apex were combined into one group: $* * * p<5 e-4 ; * * p<0.005 ; * p<0.05$; one-way ANOVA with Tukey post hoc.

ISC ROI region were analyzed. The signal-to-noise ratio was extremely high within individual ISC ROIs (see Fig. 5E), and ISC events were defined by contiguous activation of connected ISC ROIs (all 26 edges and vertices of each time point per ROI were considered; see Movie 2). IHCs were semiautomatically detected by finding local minima within 


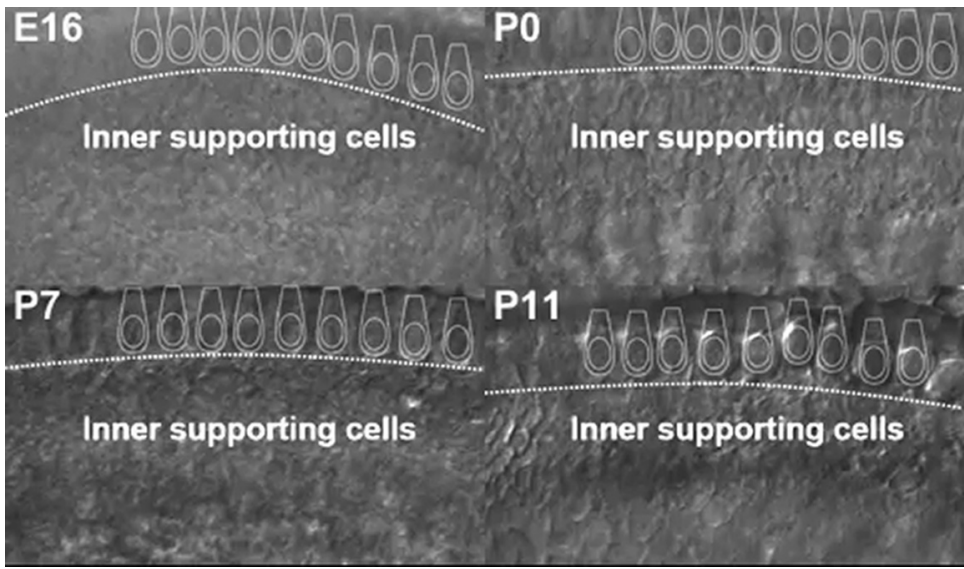

Movie 1. Delayed onset of P2ry1-dependent spontaneous crenations in ISCS. DIC imaging of spontaneous cell shrinkage events (crenation) in the cochlea across the prehearing period. Application of MRS2500 $(1 \mu \mathrm{m})$, a selective P2RY1 antagonist, is indicated in the top right corner of each video. [View online]

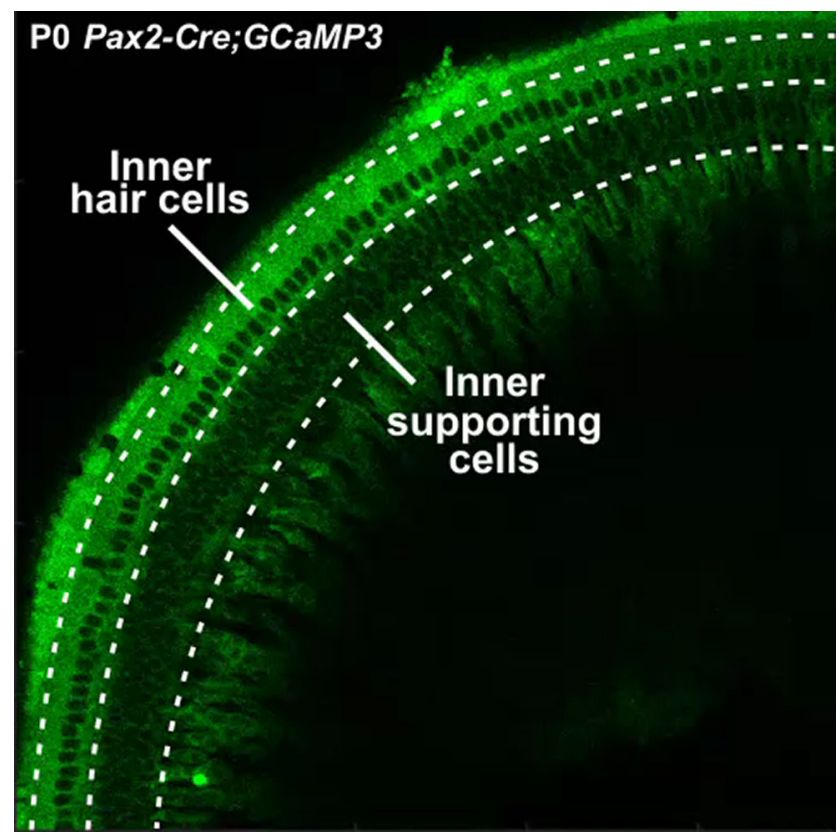

Movie 2. Grid-based analysis of $\mathrm{Ca}^{2+}$ transients. $\boldsymbol{A}$, Time-lapse imaging of isolated cochlea from PO Pax2-Cre;R26-Isl-GCaMP3 mice. White squares represent active ISCS. White dots represent active IHCs. [View online]

the IHC ROI and validated by the experimenters. Individual circular ROIs were drawn at the basal pole of each IHC. Fluorescence changes were normalized as $\Delta \mathrm{F} / \mathrm{F}_{\mathrm{o}}$ values, where $\Delta \mathrm{F}=\mathrm{F}-\mathrm{F}_{\mathrm{o}}$ and $\mathrm{F}_{\mathrm{o}}$ was defined as the fifth percentile value for each pixel. Peaks in the signals were detected in MATLAB using the built-in peak detection function (findpeaks) with a fixed value threshold criterion (median +3 SDs for ISCs and median +4 SDs for IHCs). IHC activity was considered coincident if ISC and IHC were coactive in both space and time. IHC coordinated events were defined as anytime $>3$ adjacent IHCs were coactive at the same time. Correlation coefficients reported are the 80th percentile correlation coefficient. For analysis of the extent of IHC activation, ISC events were centered around the center of mass for each event. The IHC closest to the center of mass was designated as IHC 0 and the adjacent $20 \mathrm{IHCs}$ on either side were examined. If the adjacent 20 IHCs included IHCs that were not within the imaging frame (i.e., if ISC event occurred near the edge), visible IHCs were averaged while out-of-frame IHCs were not.

For analysis of SGN signals, image stacks were imported into MATLAB where an ROI was drawn around the SGNs. A 10 pixel $\times 10$ pixel grid was imposed across the entire image, and only squares within the drawn SGN ROI region were analyzed. Fluorescence changes were normalized as $\Delta \mathrm{F} / \mathrm{F}_{\mathrm{o}}$ values, where $\Delta \mathrm{F}=\mathrm{F}-\mathrm{F}_{\mathrm{o}}$ and $\mathrm{F}_{\mathrm{o}}$ was defined as the fifth percentile value for each pixel. Peaks in the signals were detected in MATLAB using the built-in peak detection function (findpeaks) with a fixed value threshold criterion (fifth percentile value $+5 \mathrm{SDs}$ ). Active area is defined as the percentage of active ROIs (ROIs with at least 1 peak) within the drawn SGN region. Correlation coefficient was defined as the 80th percentile correlation coefficient among active ROIs only. Correlated events were defined as coincident SGN activation of 35 SGN ROIs. While this parameter (35 SGNs for a correlated event) was subjective, reanalysis of the data where this parameter was varied (down to 15 ROIs for a correlated event) revealed that relative frequencies of correlated events were preserved between conditions. Active ROI frequency, amplitude, and half-widths were calculated using only active ROIs.

Installation of cranial windows. Inhalation anesthesia was induced with vaporized isoflurane ( $4 \%$ for $5 \mathrm{~min}$, or until mice are nonresponsive to toe-pinch) and surgical plane maintained during the procedure (with $1 \%-2 \%$ isoflurane) with a stable respiration rate of 80 breaths per minute. A midline incision beginning posterior to the ears and ending just anterior to the eyes was made. Two subsequent cuts were made to remove the dorsal surface of the scalp. A headbar was secured to the head using super glue (Krazy Glue). Fascia and neck muscles overlying the interparietal bone were resected and the area bathed in sterile, HEPES-buffered ACSF that was replaced as necessary throughout the surgery. Using a $28 \mathrm{G}$ needle and microblade, the sutures circumscribing the interparietal bone were cut and removed to expose the midbrain. The dura mater was removed using fine scissors and forceps, exposing the colliculi and extensive vasculature. A $5 \mathrm{~mm}$ coverslip (CS-5R; Warner Instruments) was then placed over the craniotomy, the surrounding bone was dried using a Kimwipe, and super glue was placed along the outer edges of the coverslip for adhesion to the skull. Replacement $0.9 \% \mathrm{NaCl}$ solution was injected intraperitoneally, and a local injection of lidocaine was given to the back of the neck. Animals were weaned off isoflurane, placed under a warming lamp, and allowed to recover for a minimum of $1 \mathrm{~h}$ before imaging.

In vivo calcium imaging. After $1 \mathrm{~h}$ of postsurgical recovery from anesthesia, pups were moved into a swaddling $15 \mathrm{ml}$ conical centrifuge tube. The top half of this tube was removed to allow access to the headbar and visualization of the midbrain or midbrain and caudal part of the cortex. Pups were head-fixed and maintained at $37^{\circ} \mathrm{C}$ using a heating pad and temperature controller (TC-1000; CWE). During the experiments, pups were generally immobile; however, occasional limb and tail twitching did occur.

For wide-field epifluorescence imaging, images were captured at $10 \mathrm{~Hz}$ using a Hamamatsu ORCA-Flash4.0 LT digital CMOS camera attached to a Carl Zeiss Axio Zoom.V16 stereo zoom microscope. A $4 \times 4 \mathrm{~mm}$ FOV was illuminated continuously with a mercury lamp (Carl Zeiss Illuminator $\mathrm{HXP} 20^{\circ} \mathrm{C}$ ) and visualized through a $1 \times$ PlanNeoFluar $\mathrm{Z} 1.0 \times$ objective at $17 \times$ zoom. Images were captured at a resolution of $512 \times 512$ pixels ( 16 bit pixel depth) after $2 \times 2$ binning to increase sensitivity. Each recording consisted of uninterrupted acquisition over $30 \mathrm{~min}$ or $40 \mathrm{~min}$ if injected with pharmacological agents.

Catheterization of animals for in vivo imaging. After induction of anesthesia and before installing the cranial window, a catheter was placed in the intraperitoneal space of neonatal mouse pups. A $24 \mathrm{G}$ needle was used to puncture the peritoneum, and a small-diameter catheter (SAI Infusion Technologies, MIT-01) was placed. A small drop of Vetbond secured the catheter to the pup's belly. Installation of cranial window proceeded as described above.

Imaging sessions consisted of $15 \mathrm{~min}$ of baseline activity measurements, followed by a slow push of either $50 \mu \mathrm{l}$ of sham (5\% mannitol solution) or MRS2500 solution (500 $\mu \mathrm{M}$ in 5\% mannitol solution). 


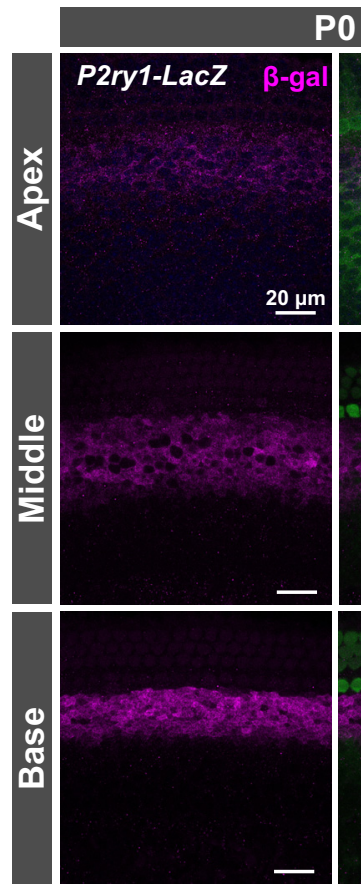

PO
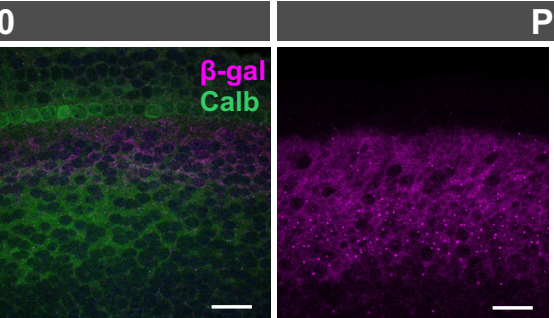

P7
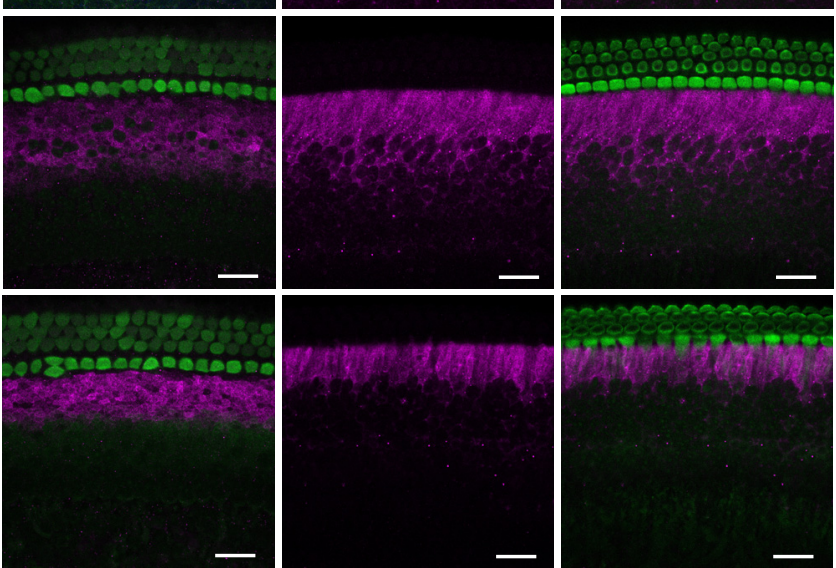
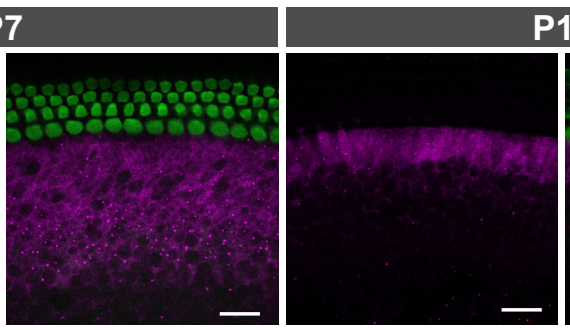

P11

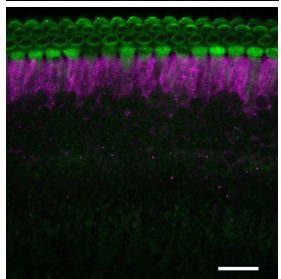

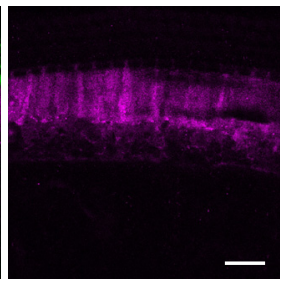
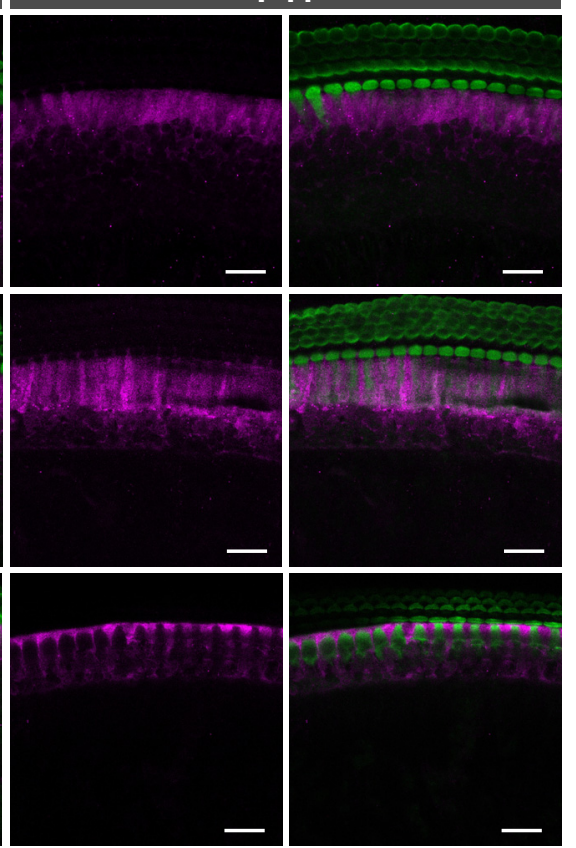

Figure 2. P2ry1 promoter activity in ISCs throughout the prehearing period. Immunostaining of cochlear sections from P0-P11 P2ry1-LacZ mice for $\beta$-galactosidase (rabbit anti- $\beta$-galactosidase, Sanes laboratory) and calbindin (goat anti-calbindin, Santa Cruz Biotechnology) for labeling of hair cells.

Imaging was continuous throughout and $45 \mathrm{~min}$ of activity total was collected. No discernable diminishment of activity was observed in sham animals.

In vivo image processing. For in vivo wide-field imaging, raw images were imported into the MATLAB environment and corrected for photobleaching by fitting a single exponential to the fluorescence decay and subtracting this component from the signal. Intensities were normalized as $\Delta \mathrm{F} / \mathrm{F}_{\mathrm{o}}$ values, where $\Delta \mathrm{F}=\mathrm{F}-\mathrm{F}_{\mathrm{o}}$ and $\mathrm{F}_{\mathrm{o}}$ was defined as the fifth percentile value for each pixel. Ovoid ROIs encompassing the entire left and right inferior colliculi (IC) were drawn. Across all conditions, the size of the ROIs was invariant. However, because of small differences in the imaging field between animals, the ROIs were placed manually for each imaging session. Peaks in the signals were detected in MATLAB using the built-in peak detection function (findpeaks) using a fixed value threshold criterion; because fluorescence values were normalized, this threshold was fixed across conditions $\left(2 \% \Delta \mathrm{F} / \mathrm{F}_{\mathrm{o}}\right)$. Occasionally, large events in the cortex or superior colliculus would result in detectable fluorescence increases in the IC. These events broadly activated the entire surface of the IC and did not exhibit the same spatially confined characteristics as events driven by the periphery. These events were not included in the analysis.

L-R correlations were calculated from $\Delta \mathrm{F} / \mathrm{F}_{\mathrm{o}}$ traces of activity from left and right IC using the corr function in MATLAB; two-thirds max intensity area was calculated at the peak of each event as the number of pixels with $\Delta \mathrm{F} / \mathrm{F}_{\mathrm{o}}$ values above two-thirds of the maximum pixel fluorescence.

Analysis of retinal wave activity in the superior colliculus. ROIs $(200 \times 150$ pixels $)$ were placed over each lobe of the superior colliculus
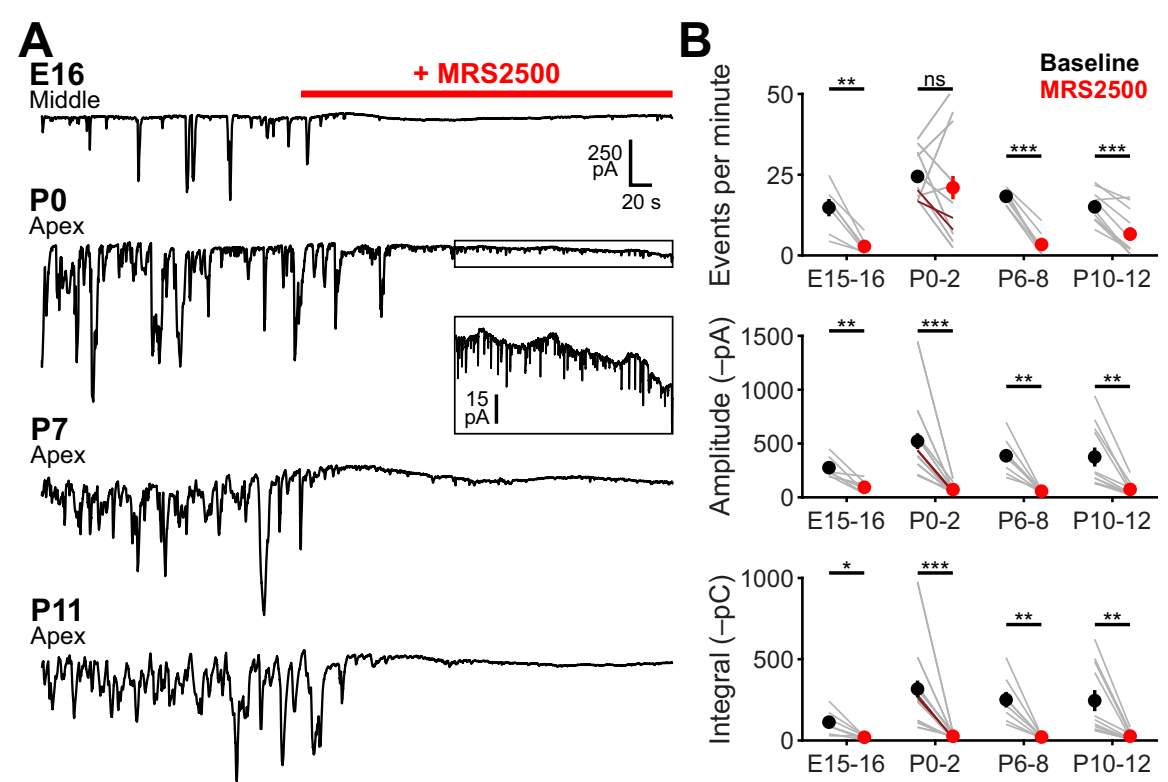

Figure 3. P2RY1 mediates supporting cell spontaneous currents in the cochlea throughout the prehearing period. $\boldsymbol{A}$, Spontaneous inward currents recorded from ISCS (E16: middle, P0-P11: apical) before and during application of MRS2500 (1 $\mu \mathrm{m})$ at different developmental ages. Recordings were made at near physiological temperature $\left(32^{\circ} \mathrm{C}-34^{\circ} \mathrm{C}\right)$. Box in P0 recording is expanded vertically below the recording. $B$, Quantification of ISC spontaneous current frequency, amplitude, and integral (charge transfer) before and after application of MRS2500. $n=7$ E16-17 ISCS, 7 cochleae from 7 mice, $n=13$ P0-2 ISCS ( $n=11$ apical in gray, $n=2$ basal in dark red), 11 cochleae from 8 mice, $n=8$ P7-8 ISCS, 8 cochleae from 8 mice, and $n=11$ cochleae from 8 mice. $* * * p<5 \mathrm{e}-4 ; * * p<0.005 ; * p<0.05$; Student's paired $t$ test with Bonferroni correction.

and downsampled by a factor of 5 . Signals were normalized as $\Delta F / F_{o}$ values, where $\Delta \mathrm{F}=\mathrm{F}-\mathrm{F}_{\mathrm{o}}$ and $\mathrm{F}_{\mathrm{o}}$ was defined as the fifth percentile value for each pixel. In order to eliminate periodic whole-sample increases in fluorescence, the mean intensity of all pixels was subtracted from each individual pixel. Following this, pixels were considered active if they exceeded the mean +3 SDs. For each point in time, the number of active pixels was summed. Retinal waves were defined as prolonged periods 
A
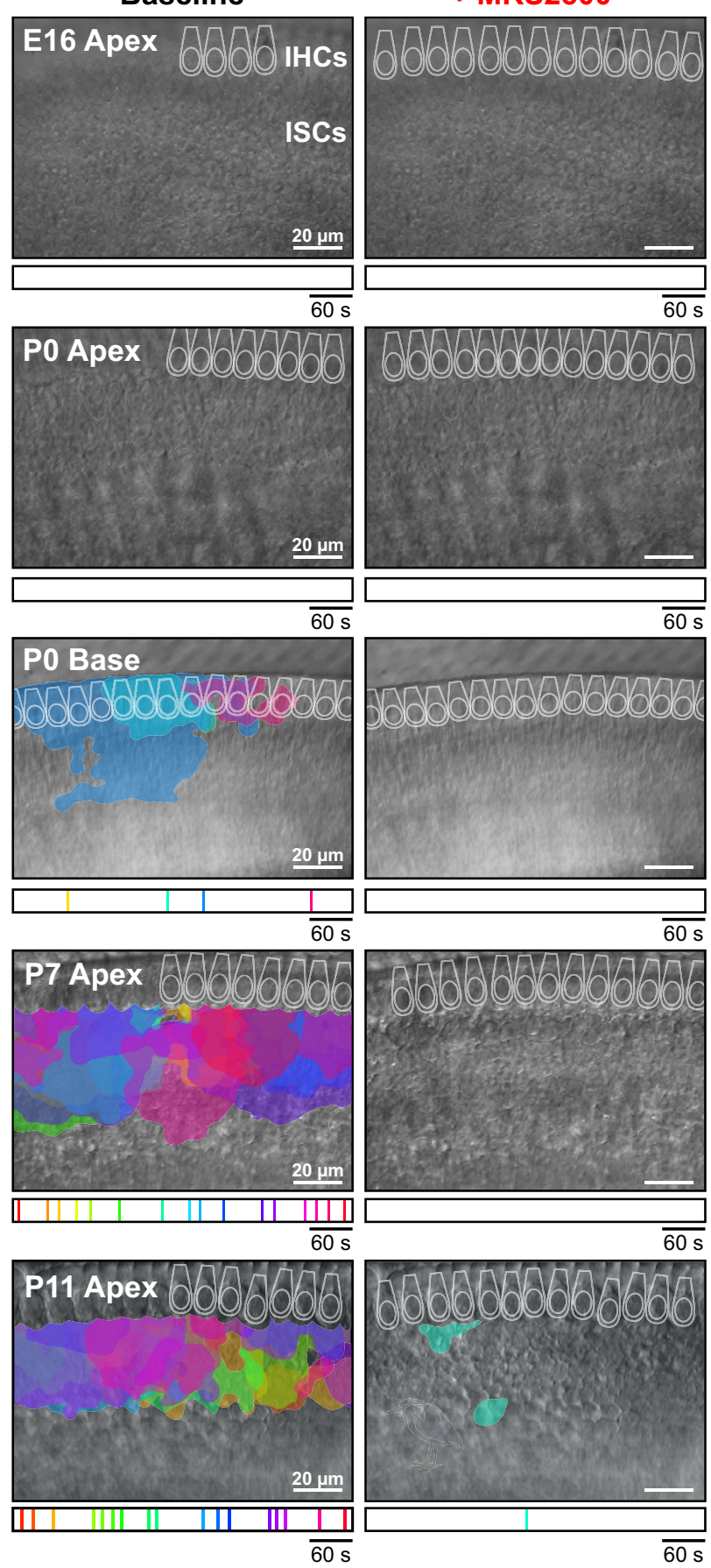

B

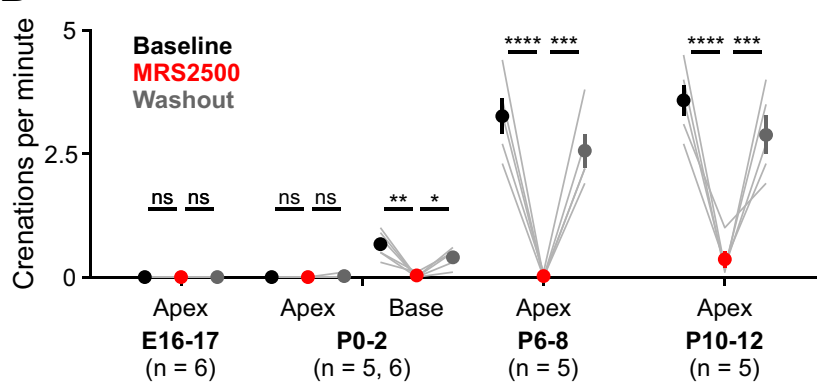

Figure 4. Delayed onset of P2RY1-dependent spontaneous crenations in ISCs. $\boldsymbol{A}$, Intrinsic optical imaging performed before and after application of the P2RY1 antagonist, MRS2500 (1 $\mu \mathrm{m})$. Detected crenations are outlined in colors based on time of occurrence as indicated
$(>1 \mathrm{~s})$, where $>5$ pixels were active simultaneously. Retinal wave durations were defined as the total continuous amount of time that $>5$ pixels were active.

Experimental design and statistical analysis. All statistics were performed in the MATLAB programming environment. All statistical details, including the exact value of $n$, what $n$ represents, and which statistical test was performed, can be found in the figure legends. To achieve statistical power of 0.8 with a $30 \%$ effect size with means and SDs similar to those observed in previous studies (Tritsch et al., 2007, their Fig. 1E; H. C. Wang et al., 2015, their Figs. $1 B, 3 D$ ), power calculations indicated that 7 animals in each condition were necessary $\left(\mu_{1}=10, \mu_{2}=7, \sigma=2\right.$, sampling ratio $=1)$. While this number was used as a guide, power calculations were not explicitly performed before each experiment; many experiments had much larger effect sizes, and sample sizes were adjusted accordingly. For transparency, all individual data points are included in the figures. Data are presented as mean \pm SEM. Because the main comparison between conditions was the mean, the SEM is displayed to highlight the dispersion of sample means around the population mean. All datasets were tested for Gaussian normality using the D'Agostino's $\mathrm{K}^{2}$ test. For single comparisons, significance was defined as $p \leq 0.05$. When multiple comparisons were made, the Benjamini-Hochberg or Bonferroni correction was used to adjust $p$ values accordingly to lower the probability of Type I errors. For multiple condition datasets, one-way ANOVAs were used, followed by Tukey's multiple comparison tests. Code and processed data used for analysis are available on Github (https:// github.com/tbabola/2020_Babola_JNeuro).

\section{Results}

Spontaneous electrical activity of ISCs emerges before birth In the developing mammalian cochlea, supporting cells within Kölliker's organ spontaneously release ATP, initiating a purinergic signaling cascade that releases $\mathrm{Ca}^{2+}$ from intracellular stores and activates TMEM16A, a Ca ${ }^{2+}$-activated $\mathrm{Cl}^{-}$channel (H. C. Wang et al., 2015; Babola et al., 2020). Efflux of $\mathrm{Cl}^{-}$ions draws positive $\mathrm{K}^{+}$ions into the extracellular space, producing a temporary osmotic gradient that draws water into the extracellular space. Because of extensive gap-junction coupling between ISCs, activation of these purinergic pathways induces large currents and cellular shrinkage (crenation) among groups of these cells. Local increases in extracellular $\mathrm{K}^{+}$depolarize nearby IHCs, resulting in bursts of action potentials, glutamate release, and activation of AMPARs and NMDARs on postsynaptic SGNs (Zhang-Hooks et al., 2016); thus, unlike hearing, this electrical activity does not require activation of mechanotransduction channels (Sun et al., 2018). Spontaneous inward currents in ISCs are present from birth, but little is known about when this activity emerges (Tritsch and Bergles, 2010; H. C. Wang et al., 2015; Zhang-Hooks et al., 2016). To determine the onset of spontaneous ISC currents, we made whole-cell voltage-clamp recordings from ISCs in cochleae acutely isolated from embryonic day 14-16 (E14-E16) mouse pups (Fig. 1A), a developmental period characterized by basal to apical differentiation of inner and outer hair cells (Chen et al., 2002). Recordings from ISCs in the apical region of the cochleae revealed no discernable spontaneous currents ( 6 of 6 cochleae; Fig. $1 B$ ). In contrast, large spontaneous currents were observed in most cochleae in the

\section{$\leftarrow$}

by timeline below image. Imaging was performed near physiological temperature $\left(32^{\circ} \mathrm{C}-34^{\circ}\right.$ C). $\boldsymbol{B}$, Plot of crenation frequency and area before and after application of MRS2500. $n=6$ E16-17 videos, 6 cochleae from 6 mice, $n=5$ apical P0-2 videos, 5 cochleae from 5 mice, $n=6$ basal P0-2 videos, 6 cochleae from 6 mice, $n=5$ apical P6-8 videos, 5 cochleae from 5 mice, and $n=5$ apical P10-12 videos, 5 cochleae from 5 mice. $* * * * p<5 \mathrm{e}-5$; $* * * p<5$ e-4; one-way ANOVA with Tukey post hoc. $* p<0.05, * * p<0.005$. 

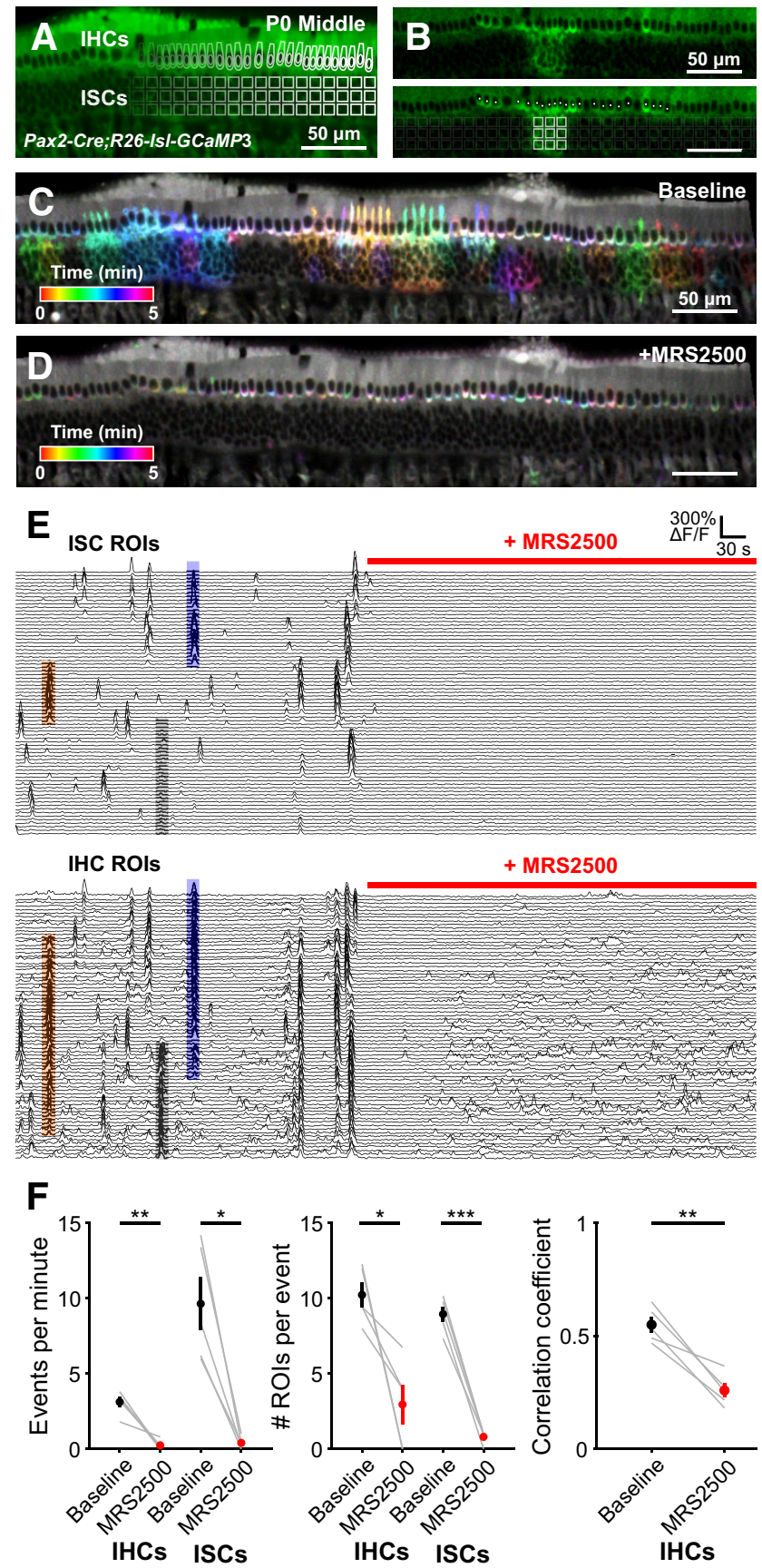

Figure 5. Correlated activation of IHCS and ISCS requires P2RY1 signaling. $A$, Image of an excised cochlea (middle turn) from a PO Pax2-Cre;R26-Isl-GCaMP3 mouse. For analysis of time-lapse imaging, a grid of square ROls was placed over the ISCs and single ROls were drawn for each IHC. Imaging was performed at near physiological temperature $\left(32^{\circ} \mathrm{C}-34^{\circ} \mathrm{C}\right)$. $B$, Exemplar $\mathrm{Ca}^{2+}$ transient in ISCS and simultaneous activation of multiple IHC. Bottom, Circles represent active IHCs. White squares represent active ISCS. C, $\mathrm{Ca}^{2+}$ transients in control (baseline) conditions colored based on time of occurrence. $\boldsymbol{D}, \mathrm{Ca}^{2+}$ transients with P2RY1 inhibited (MRS2500, $1 \mu \mathrm{m}$ ) conditions colored based on time of occurrence. $\boldsymbol{E}$, Individual ROI traces for ISCS (top, 100 randomly selected) and IHCS (bottom, all shown). Colored boxes are examples of coordinated activity of ISCs and IHCS. The number of IHCS activated can extend far beyond area of ISC activation (see Fig. 6). Gray box represents IHC activation on the edge of the frame with no ISC activation, likely caused by an out-of-frame ISC event. $\boldsymbol{F}$, Quantification of coordinated event frequency, number of ROls per coordinated event, and the correlation coefficient before and after application of MRS2500. $n=5$ cochleae from 3 mice. $* * * p<5 \mathrm{e}-4 ; * * p<0.005 ; * p<0.05$; paired $t$ test with BenjaminiHochberg adjustment.

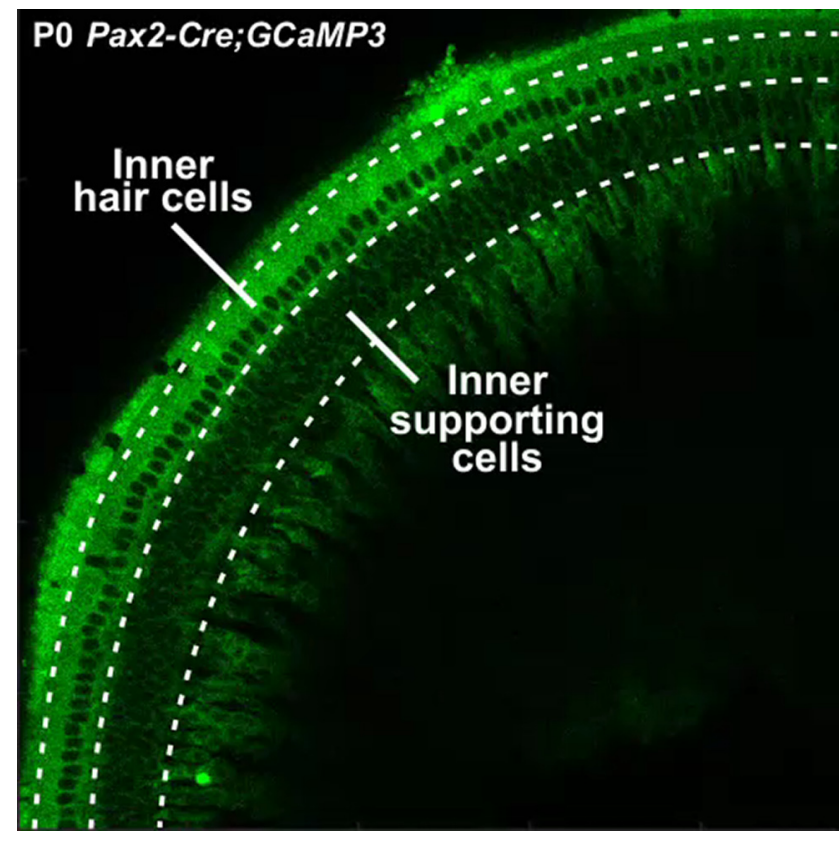

Movie 3. Correlated activation of IHCS and ISCS requires P2RY1 signaling. A, Time-lapse imaging of isolated middle sections of cochlea from P0 Pax2-Cre;R26-Isl-GCaMP3 mice. Application of MRS2500 $(1 \mu \mathrm{M})$ is indicated in the top right corner of each video. [View online]

basal region (3 of 3 cochleae at E16 and 1 of 3 cochleae at E14). After birth, spontaneous inward currents were observed in apical and basal ISCs throughout the early postnatal period (Fig. 1C), consistent with previous observations (Tritsch et al., 2007; Tritsch and Bergles, 2010). At P0, currents in apical ISCs were more frequent ( $24 \pm 2$ vs $1 \pm 1$ events per minute; one-way ANOVA, $F_{(5,42)}=24.95, p=6 \mathrm{e}-9$; Tukey HSD, $\left.p=2 \mathrm{e}-8\right)$, larger in amplitude $(522 \pm 100$ vs $44 \pm 10 \mathrm{pA}$; one-way ANOVA, $F_{(5,42)}=3.55, p=0.013$; Tukey HSD, $\left.p=0.010\right)$, and carried more charge per second (integral; $320 \pm 80$ vs $15 \pm 5 \mathrm{pC}$; one-way ANOVA, $F_{(5,42)}=0.018$; Tukey HSD, $p=0.019$ ) than in embryonic ISCs (Fig. 1D). Similar increases in frequency occurred in basal ISCs at P0 (14 \pm 2 vs $5 \pm 1$ events per minute; Tukey HSD, $p=6 \mathrm{e}-9$ ), with event amplitudes and integrals trending larger than in embryonic ISCs, but not reaching statistical significance (Tukey HSD, $p=0.28$ and $p=0.59$ ). While there was a progressive decline in average frequency, amplitude, and integral postnatally up to hearing onset $(\sim \mathrm{P} 12)$, only decreases in event frequency were statistically significant (P0 vs P12, Tukey HSD, $p=4.4 \mathrm{e}-4$; Fig. $1 D)$. The lack of spontaneous currents in apical ISCs at embryonic ages may reflect reduced gap junctional coupling, which would prevent detection of currents that arise in distant cells. However, the membrane resistances of apical ISCs were consistently low (Fig. 1D; E14-E16: $11 \pm 2 \mathrm{~m} \Omega$, P0-2: $11 \pm 4 \mathrm{~m} \Omega$, P7-8: $9 \pm 3 \mathrm{~m} \Omega, \mathrm{P} 10-12: 14 \pm 4$ $\mathrm{M} \Omega$; one-way ANOVA, $\left.F_{(5,42)}=2.02, p=0.09\right)$. As membrane resistance is determined primarily by cell-to-cell coupling (Jagger and Forge, 2006), these results suggest that gap junctional coupling among ISCs across this developmental period is similar (Jagger and Forge, 2006; Kamiya et al., 2014). Together, these data indicate that spontaneous currents emerge in ISCs during the late embryonic period in a basal to apical gradient, matching the progression of hair cell maturation. 
A

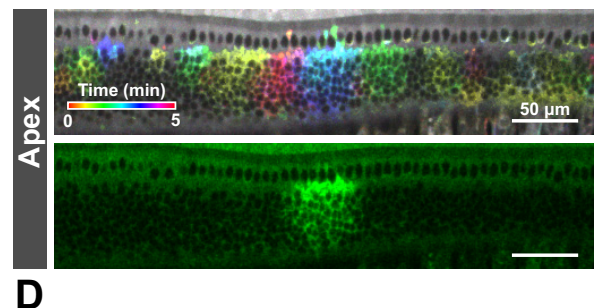

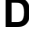

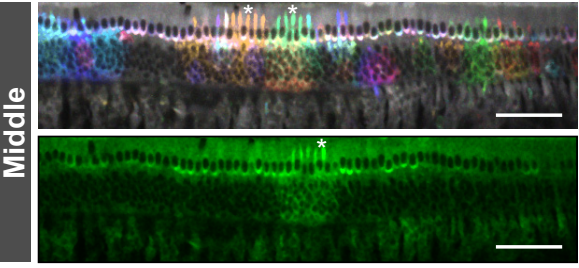

E

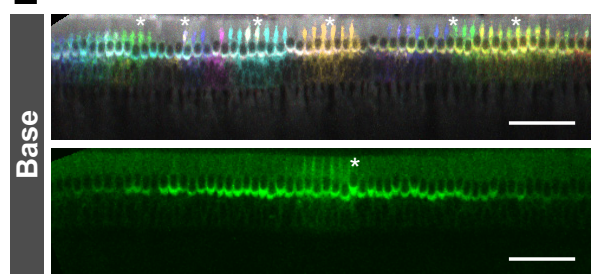

B
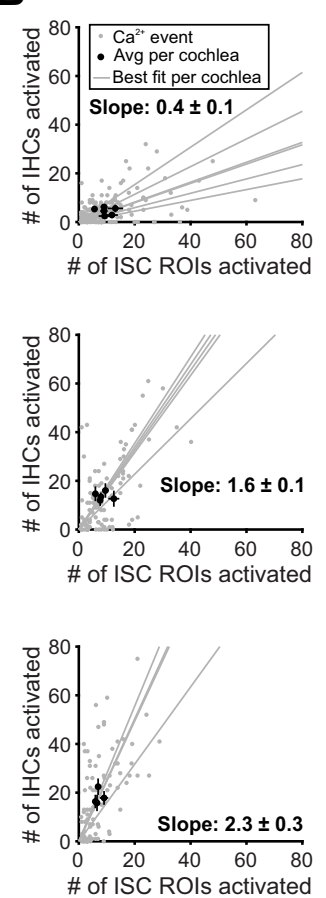

C
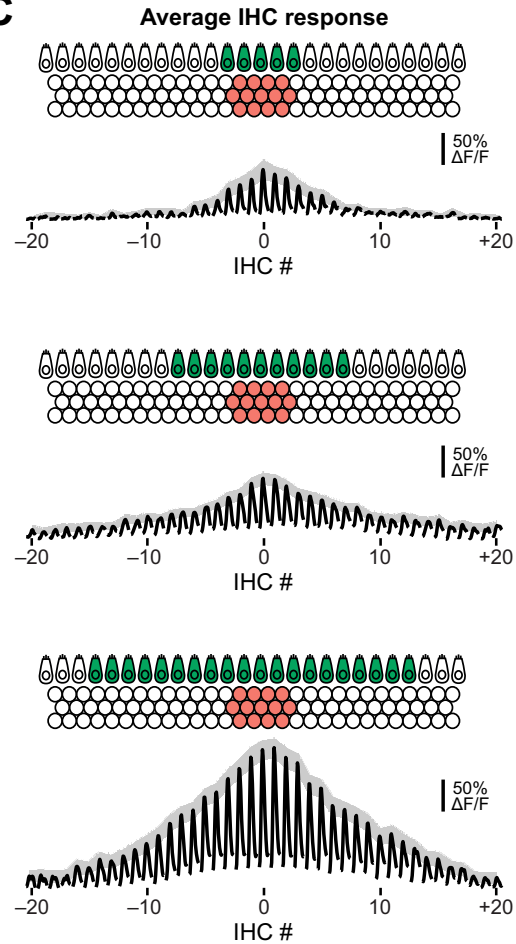

Figure 6. Tonotopic differences in extent of IHC activation at early developmental time points. $\boldsymbol{A}$, Images of $\mathrm{Ca}^{2+}$ transients colored based on time of occurrence (top) and exemplar $\mathrm{Ca}^{2+}$ transient (bottom) in the apical portions of cochleae isolated from PO Pax2-Cre;R26-Isl-GCaMP3 mice. Imaging was performed at near physiological temperature ( $\left.32^{\circ} \mathrm{C}-34^{\circ} \mathrm{C}\right)$. $\boldsymbol{B}$, Plot of number of IHCs activated as a function of the number of ISC ROIs activated for apical (top, $n=6$ cochleae) regions of the cochlea. Gray dots represent individual $\mathrm{Ca}^{2+}$ transients. Gray lines indicate linear best fits for each cochlea. Black dots and lines indicate the mean event size \pm SEM for each cochlea. Calculated slope is the mean \pm SEM of the best fit lines. C, Schematic and average IHC response for aligned ISC events in the apical (top), middle (middle), and basal (bottom) regions of the cochlea. Black traces represent the average IHC responses to an ISC event with centroid closest to center IHC (IHC at 0). Gray shaded region represents SEM for the peak of each IHC. D, E, Similar to $A-C$, but for middle ( $n=5$ cochleae) and basal ( $n=4$ cochleae) portions of the cochlea. Asterisks indicate activated inner phalangeal cells.

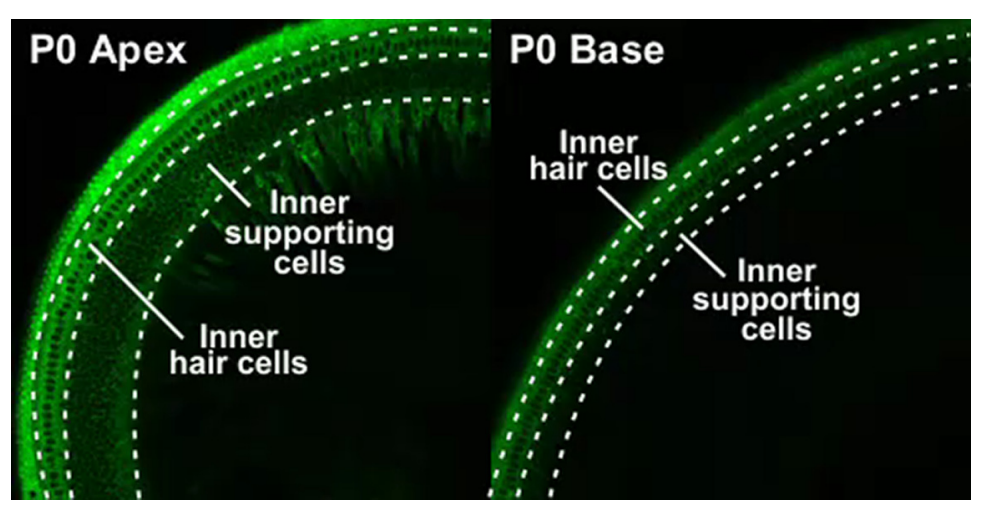

Movie 4. Tonotopic differences in extent of IHC activation at early developmental time points. A, Timelapse imaging of isolated apical and basal sections of cochlea from Pax2-Cre;R26-Isl-GCaMP3 mice (P0). [View online]

\section{Supporting cell spontaneous currents and crenation are} mediated by P2RY 1 throughout the prehearing period Spontaneous currents in ISCs require activation of purinergic receptors between birth and shortly after hearing onset, when Kölliker's organ recedes (Tritsch and Bergles, 2010). Recently, the $\mathrm{G}_{\mathrm{q}}$-coupled P2Y1 receptor (P2RY1) was identified as the primary purinergic autoreceptor mediating spontaneous currents in ISCs after the first postnatal week. Gene expression studies revealed that P2ry1 is expressed at much higher levels $(>100$ fold) than any other P2Y receptor in the cochleae, even at early embryonic ages (Scheffer et al., 2015; Kolla et al., 2020), suggesting that this receptor may initiate spontaneous currents throughout development. However, the presence of $\mathrm{Ca}^{2+}$-permeable ionotropic (P2X2/4) and $\mathrm{G}_{\mathrm{q}}$-coupled metabotropic receptors (P2RY2/4/6) in the cochlea (Huang et al., 2010; Housley et al., 2013; Kolla et al., 2020) indicate that alternative pathways could also contribute to spontaneous activity during this period, depending on the amount, location, and kinetics of ATP release, as well as the presence and activity of extracellular nucleotidases. To define the dynamics of P2RY1 expression during cochlear development, we isolated cochleae from P2ry1-LacZ reporter mice and performed immunostaining for $\beta$-galactosidase at different developmental ages (Fig. 2). Immunofluorescence within Kölliker's organ and along the entire length of the cochlea was detected across all postnatal time points (P0, P7, and P11). At later stages of development, $\beta$-galactosidase immunofluorescence was observed in interdigitating phalangeal cells, primarily within the base at P7 and by P11 along the entire length of the cochlea (Fig. 2). These data indicate that P2RY1 promoter activity is localized to ISCs throughout the prehearing period, providing the means to express P2RY1 and detect ATP release from these cells.

To determine whether P2RY1 mediates spontaneous currents in ISCs across this developmental period, we examined the sensitivity of these responses to the specific P2RY1 antagonist, MRS2500 (Houston et al., 2006), which displays no obvious offtarget effects in cochleae isolated from P2RY1 KO mice (Babola 

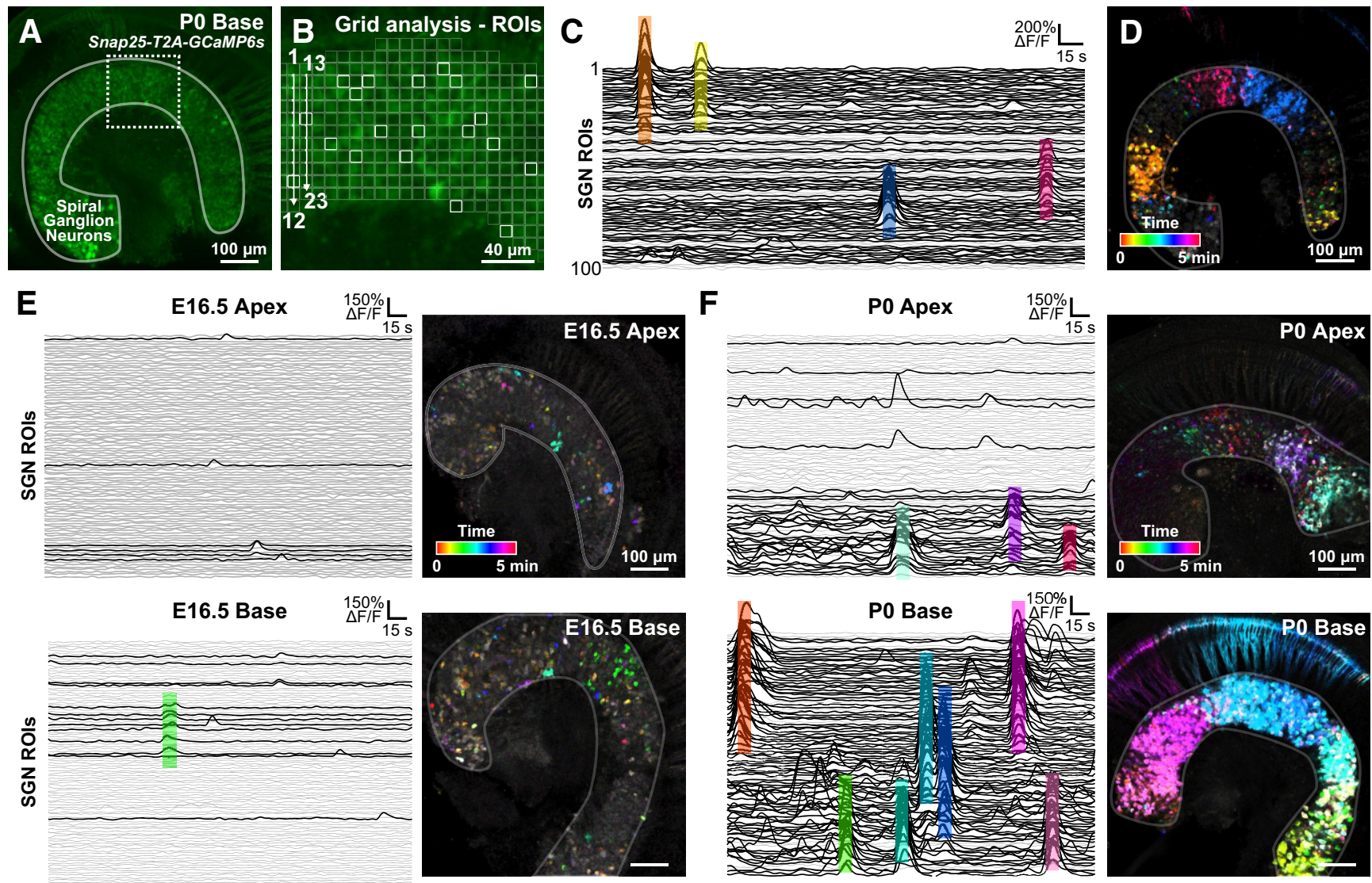

G
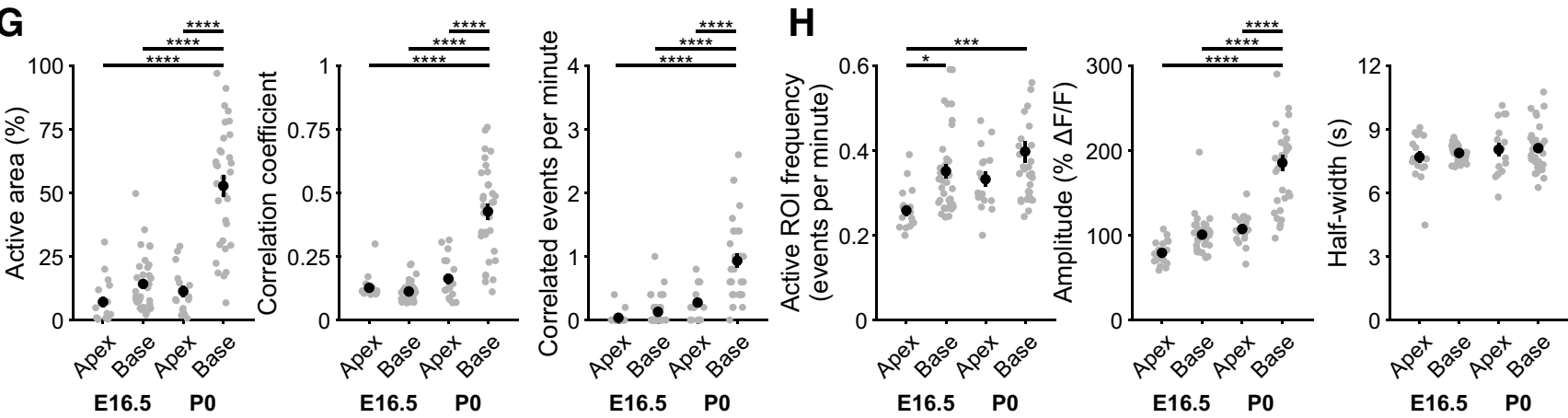

Figure 7. Tonotopic differences in extent of SGN activation at early developmental time points. A, Image of an excised basal portion of cochlea from a P0 Snap25-T2A-GCaMP6s mouse, which expresses GCaMP6s in SGNs. Dotted line indicates region shown in $\boldsymbol{B}$. $\boldsymbol{B}$, For analysis of time-lapse imaging, a grid of square ROls was placed over SGNs. ROls were numbered top to bottom, then left to right. All ROls were analyzed, but only random ROls were chosen to display in figures (white squares). Imaging was performed at room temperature $\left(\sim 25^{\circ} \mathrm{C}\right)$. $C$, Individual ROI traces for SGNs (100 randomly selected). Colored boxes are examples of SGN coordinated activity that align with time-color representation in $\boldsymbol{D}$. Black traces represent ROls with at least one detected peak (fifth percentile value \pm 5 SDs). Gray traces represent ROIs with no detected peaks. D, SGN $\mathrm{Ca}^{2+}$ transients colored based on time of occurrence. $\boldsymbol{E}$, Individual ROI traces and time-color representation of $\mathrm{Ca}^{2+}$ transients in E16.5 apical (top) and basal (bottom) regions of the cochlea. Black traces represent ROls with at least one detected peak (fifth percentile value \pm 5 SDs). Gray traces represent ROls with no detected peaks. $\boldsymbol{F}$, Similar to $\boldsymbol{E}$, but in P0 apical (top) and basal (bottom) regions of the cochlea. $\boldsymbol{G}$, Quantification of active area (percentage of ROls with at least one detected peak), correlation coefficient (80th percentile), and correlated events per minute in E16.5 and PO cochleae. $n=17$ E16.5 apical portions from 9 mice, $n=34$ E16.5 basal portions from 17 mice, $n=16 \mathrm{PO}$ apical portions from 8 mice, and $n=32 \mathrm{PO}$ basal portions from 16 mice. $* * * * p<5$ e-5; one-way ANOVA with Tukey post hoc. $\boldsymbol{H}$, Quantification of frequency, amplitude, and duration of $\mathrm{Ca}^{2+}$ transients calculated from individual active ROls. $n$ values are reported in $\mathbf{G} . * * * * p<5 \mathrm{e}-5$; $* * * p<5 \mathrm{e}-4$; $* p<0.05$; one-way ANOVA with Tukey post hoc. All comparisons not indicated were not statistically significant.

et al., 2020). At the earliest time points exhibiting robust spontaneous activity (E16-17), acute inhibition of P2RY1 with MRS2500 $(1 \mu \mathrm{M})$ dramatically reduced the frequency (baseline: $15 \pm 3$, MRS2500: $3 \pm 1$ events per minute; Student's $t$ test with Bonferroni correction, $t_{(6)}=5.36, p=0.0017$ ), amplitude (baseline: $280 \pm 40$, MRS2500: $94 \pm 20 \mathrm{pA}$; Student's $t$ test with Bonferroni correction, $\left.t_{(6)}=4.37, p=0.0047\right)$, and total charge transfer of spontaneous inward currents (baseline: $110 \pm 30$, MRS2500: $20 \pm 4$ pC; Student's $t$ test with Bonferroni correction, $t_{(6)}=3.43, p=0.0140$ ) (Fig. $\left.3 A, B\right)$. Spontaneous currents were also largely inhibited by MRS2500 at P0, P7, and just before hearing onset (P10-12); only small-amplitude currents persisted in the presence of this antagonist (Fig. $3 A$, inset), consistent with previous observations of residual nonpurinergic mediated currents in these cells (Babola et al., 2020).

The efflux of $\mathrm{K}^{+}$and $\mathrm{Cl}^{-}$following purinergic receptor activation induces osmotic shrinkage (crenation) of ISCs through movement of water down its osmotic gradient (Tritsch et al., 


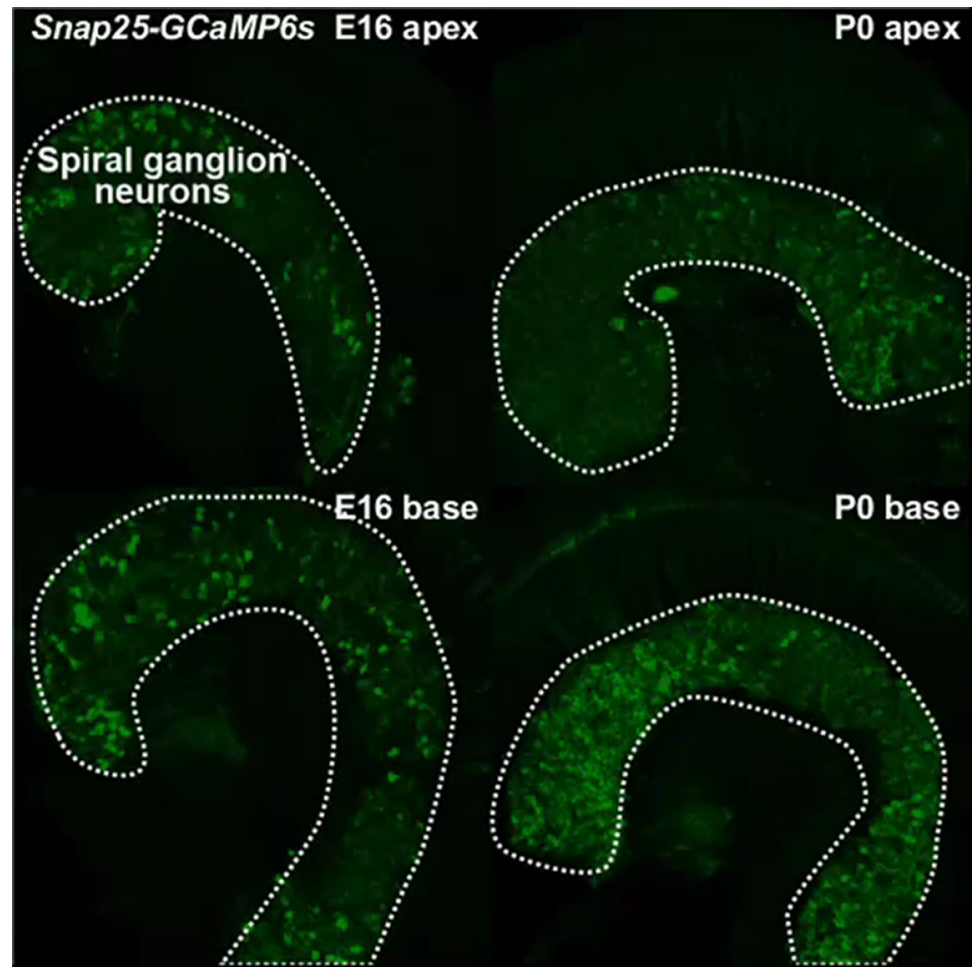

Movie 5. Tonotopic differences in extent of SGN activation at early developmental time points. A, Timelapse imaging of SGN activity in isolated apical and basal sections of cochlea from E16.5 and P0 Snap25T2A-GCaMP6s mice. [View online]

2007; H. C. Wang et al., 2015). Previous studies revealed that crenations are small and infrequent at birth in apical portions of the cochlea but rapidly increase in frequency and size over the first postnatal week (Tritsch and Bergles, 2010). To determine whether P2RY1 mediates cellular crenation throughout development, we monitored crenations in acutely isolated cochleae using DIC before and after application of the P2RY1 antagonist, MRS2500. Crenations were absent in embryonic and P0 apical sections (Fig. 4A), and application of MRS2500 at these ages resulted in no change in the optical properties of the tissue (Fig. $4 B$; Movie 1). In contrast, crenations were present at low frequencies $(0.7 \pm 0.1$ crenations per minute) in basal sections of $\mathrm{P} 0$ cochleae and robust in P7 and P11 apical sections (3.3 \pm 0.4 and $3.6 \pm 0.3$ crenations per minute, respectively), with the majority of events occurring near the medial edge of IHCs (Fig. 4B). Crenation in these preparations was reversibly blocked by MRS2500 (Fig. 4B; Movie 1). Together, these results indicate that P2RY1 induces ISC spontaneous currents and crenations throughout the prehearing period.

\section{Correlated activation of IHCs requires activation of ISC P2Y1 receptors}

The rapid increase in extracellular $\mathrm{K}^{+}$following activation of ISC purinergic autoreceptors depolarizes nearby IHCs, resulting in high-frequency burst firing that triggers glutamate release and subsequent activation of SGNs. Previous studies revealed that activation of P2RY1 autoreceptors is required to induce coordinated activation of groups of ISCs and nearby IHCs after the first postnatal week (Babola et al., 2020). To evaluate whether P2RY1 initiates coordinated activity patterns in ISCs and IHCs at earlier developmental time points, we monitored large-scale activity patterns in excised cochleae from P0 Pax2-Cre;R26-lsl-GCaMP3 mice, which express GCaMP3 in nearly all cells of the cochlea
(Fig. $5 A$ ). We quantified activity patterns by placing a grid composed of square ROIs $(10 \times 10$ pixels $)$ over the ISC region and circular ROIs around the basal pole of each IHC, where $\mathrm{Ca}_{\mathrm{v}} 1.3 \mathrm{Ca}^{2+}$ channels enable depolarization-induced $\mathrm{Ca}^{2+}$ influx (Fig. 5A,B) (Brandt et al., 2003; Zampini et al., 2014). Time-lapse imaging revealed robust spontaneous $\mathrm{Ca}^{2+}$ transients in ISCs and concurrent activation of nearby IHCs (Fig. 5C,E; Movie 2). These coordinated transients were abolished following inhibition of P2RY1 with MRS2500 (Fig. 5D,E; Movie 3). At later postnatal ages, persistent inhibition of P2RY1 results in a gradual increase in noncorrelated activity in IHCs because of an accumulation of extracellular $\mathrm{K}^{+}$(Babola et al., 2020). Consistent with this finding, noncorrelated IHC activity also emerged after prolonged P2RY1 inhibition in P0 cochleae (Fig. 5D-F). These data indicate that early coordinated activation of ISCs and IHCs also requires activation of P2RY1 signaling pathways.

The early postnatal period is defined by transformation of Kölliker's organ into the inner sulcus (Hinojosa, 1977) and rapid changes in the electrophysiological properties of IHCs, both of which occur in a basal to apical developmental gradient. To determine how these processes affect the activity patterns of IHCs, we assessed IHC activation in apical, middle, and basal portions of cochleae from P0 Pax2-Cre;R26lsl-GCaMP3 mice. In the apex, groups of ISCs exhibited robust coordinated $\mathrm{Ca}^{2+}$ transients that occurred along the entire length and medial-lateral portion of the imaged area (Fig. 6A). For each individual ISC event, only IHCs within the immediate area were activated $(4.5 \pm 0.6$ IHCs per ISC event; Fig. $6 A, B$; Movie 4). To determine how the area of ISC activation influences the number of IHCs activated, we examined the relationship between the number of ISC ROIs activated and the number of IHCs activated (Fig. 6B). The relationship was linear, with more IHCs active following large ISC events; however, fewer IHCs were activated in the apex $(0.4 \pm 0.1$ IHCs activated per single ISC ROI; Fig. $6 B)$. We then computationally centered each ISC event to explore how IHC activation varies as a function of distance away from the center of each ISC event (Fig. 6C). On average, IHCs in the apex were moderately activated following ISC activation, with few IHCs activated distal to the event. In contrast, in the developmentally older middle and basal portions of the cochlea, progressively more IHCs were activated on average for each ISC event $(13.8 \pm 0.7$ and $18.1 \pm 1.5$ IHCs per ISC event, respectively; Fig. $6 D, E)$. Each IHC exhibited larger $\mathrm{Ca}^{2+}$ transients (base: $310 \pm 10 \% \Delta \mathrm{F} / \mathrm{F}$ for center IHC, middle: $160 \pm 10 \% \Delta \mathrm{F} / \mathrm{F}$, and apex: $100 \pm 20 \% \Delta \mathrm{F} / \mathrm{F}$; one-way ANOVA, $F_{(2,172)}=80.95, p=2 \mathrm{e}-$ $25)$, and IHC activation extended far beyond the active ISC region (Fig. 6D,E; Movie 4). We did not observe any difference between the average number of ISCs activated per event (base: $7.1 \pm 0.6$, middle: $8.7 \pm 1.1$, and apex: $9.6 \pm 1.0$ ISCs ROIs; oneway ANOVA, $\left.F_{(2,12)}=1.45, p=0.27\right)$ or the average event amplitude (base: $82 \pm 5 \%$, middle: $106 \pm 8 \%$, apex: $101 \pm 6 \% \Delta \mathrm{F} / \mathrm{F}$; one-way ANOVA, $\left.F_{(2,12)}=2.6, p=0.11\right)$, suggesting that changes in ISC activity are not responsible for the difference in IHC activation along the tonotopic axis. However, inner phalangeal cells, which envelop IHCs, displayed prominent $\mathrm{Ca}^{2+}$ transients coincident with those in Kölliker's organ in the basal and middle turns (Fig. 6D,E, asterisks), but not in the apical turn (Fig. 6A). 

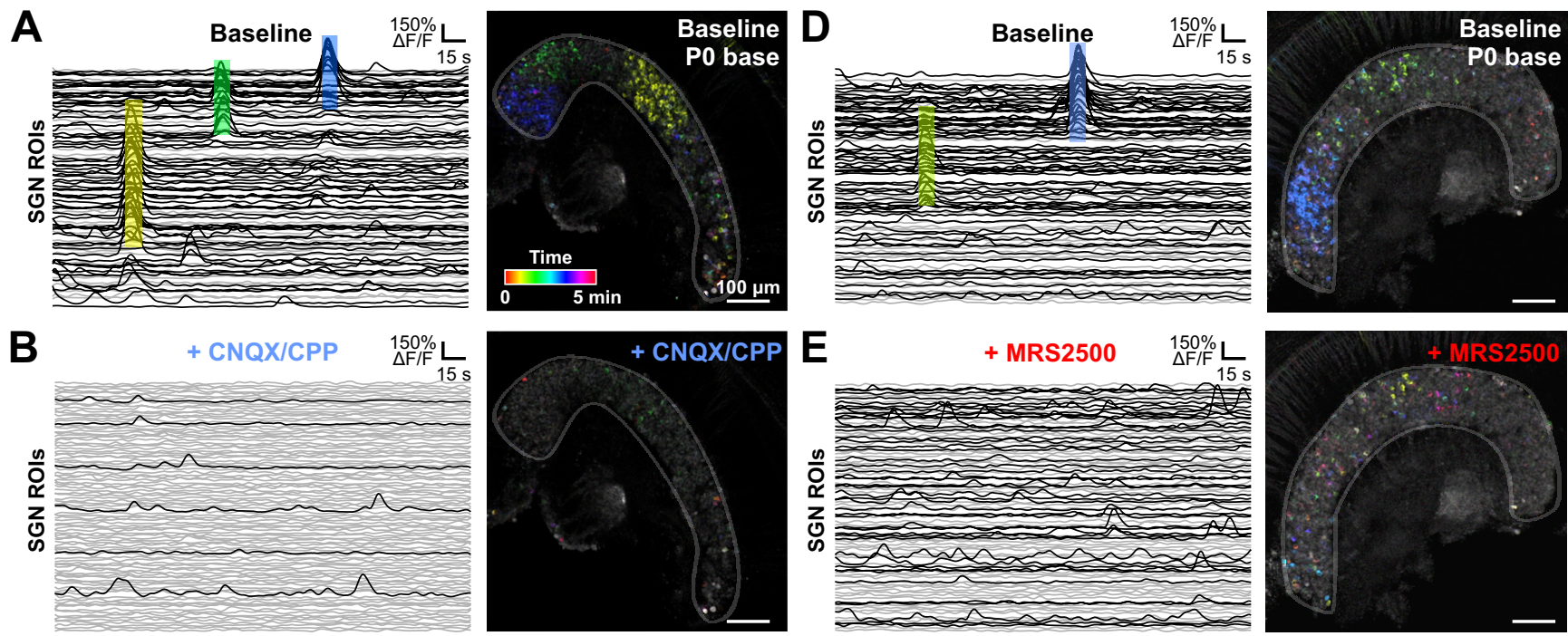

C
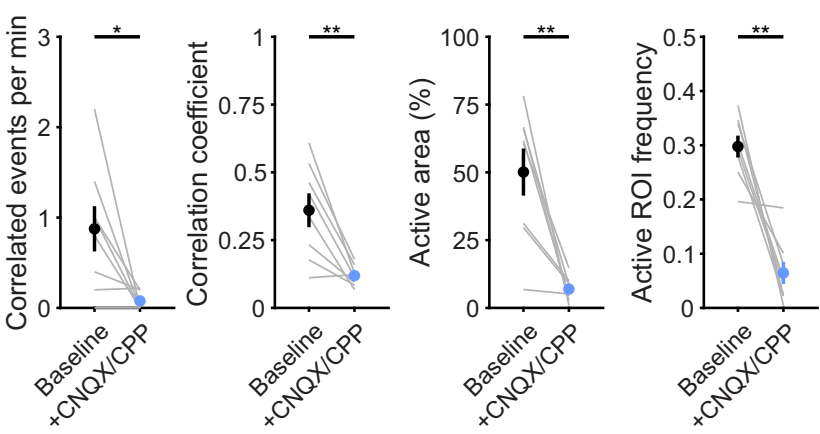
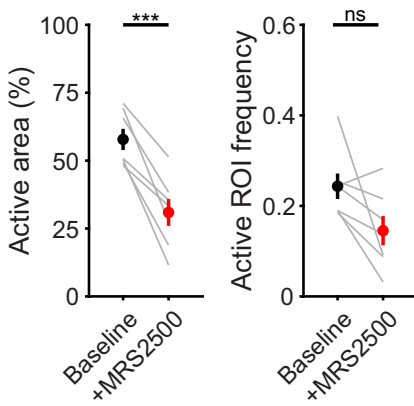

Figure 8. Correlated activation of SGNs requires P2ry1-mediated excitation of IHCs. A, Individual ROI traces and time-color representation of Ca ${ }^{2+}$ transients in control (baseline) conditions from the basal turn of cochlea isolated from a PO Snap25-T2A-GCaMP6s mouse. Colored boxes on left correspond to same colored events on right. Black traces represent ROls with at least one detected peak (fifth percentile value \pm 5 SDs). Gray traces represent ROls with no detected peaks. $\boldsymbol{B}$, Similar to $\boldsymbol{A}$, but with application of the AMPAR and NMDAR antagonists CNQX (50 $\mu$ M) and (PP (100 $\mu \mathrm{m})$. C, Quantification of correlated event frequency, correlation coefficient (80th percentile), active area (percentage of ROIs with at least one detected peak), and frequency of transients in active ROls before and after application of CNQX/CPP. $n=8$ PO basal portions from 4 mice. $* * p<0.005 ; * p<0.05$; paired $t$ test with Benjamini-Hochberg adjustment. $\boldsymbol{D}$, Similar to $\boldsymbol{A}$. $\boldsymbol{E}$, Similar to $\boldsymbol{D}$, but with bath application of P2RY1 antagonist (MRS2500, $1 \mu \mathrm{m}$ ). $\boldsymbol{F}$, Quantification of correlated event frequency, correlation coefficient (80th percentile), active area (percentage of ROIs with at least one detected peak), and frequency of transients in active ROls before and application of MRS2500. $n=7$ P0 basal portions from 4 mice. $* * * p<5$ e-4; $* * p<0.005 ; * p<0.05$; paired $t$ test with Benjamini-Hochberg adjustment.

The sparsity of $\mathrm{Ca}^{2+}$ transients in apical inner phalangeal cells suggests lower $\mathrm{Ca}^{2+}$-dependent $\mathrm{K}^{+}$extrusion near IHCs, which may contribute to the muted response of apical IHCs. Similarly, cellular crenation observed in the base, but not apex, at this age (Fig. 4A) may enhance $\mathrm{K}^{+}$diffusion and promote activation of IHCs distal to the ATP release site. Together, these data indicate that IHCs in basal portions of the cochlea are activated by ISCs at an earlier developmental stage.

\section{Correlated activation of SGNs requires P2RY1-mediated excitation of IHCs}

Previous studies indicate that burst firing of SGNs during the prehearing period requires glutamatergic synaptic excitation (Seal et al., 2008). Within apical portions of the cochlea, SGN afferent fibers extend into the newly differentiated hair cell region at E16, but SGNs do not exhibit postsynaptic densities and IHCs do not form ribbons until E18 (Michanski et al., 2019), suggesting that IHC activity may not propagate to the CNS at this stage. To determine when P2RY1-mediated currents in ISCs trigger coordinated activation of SGNs, we performed time-lapse imaging of excised cochleae from mice that expressed GCaMP6s in SGNs (Snap25-T2A-GCaMP6s mice) (Fig. 7A). Similar to the analysis of ISC activity, we placed a grid of square ROIs over
SGNs to monitor changes in fluorescence over time across the population (Fig. $7 B-D$ ). Consistent with the lack of activity in apical ISCs at E16 (Fig. 1C), SGN Ca ${ }^{2+}$ transients were infrequent and noncorrelated at this age (Fig. $7 E$ ). In the base, where ISCs exhibit robust ATP-mediated currents (Fig. 1C), SGNs were also largely silent, with some preparations (10 of 32) exhibiting infrequent, concurrent activation of groups of SGNs (Fig. 7E). However, at P0, most SGNs at the basal end of apical preparations exhibited correlated activation (10 of 16 preparations; Fig. $7 F)$, consistent with the base-to-apex emergence of activity in ISCs. Compared with E16 and P0 apical preparations, P0 basal preparations had larger average numbers of SGNs activated, higher correlations among ROIs, and more frequent correlated events (Fig. 7G; Movie 5), although no differences were observed in the duration of events (Fig. 7H). Considering only active ROIs from each preparation, transients from P0 basal preparations were more frequent than E16.5 apical preparations and were larger in amplitude than all other preparations (Fig. $7 H$ ). These data indicate that coordinated activation of SGNs emerges between E16.5 and P0 in a basal to apical developmental gradient.

Extrusion of $\mathrm{K}^{+}$into the extracellular space following P2RY1 activation nonselectively depolarizes nearby cells and their 


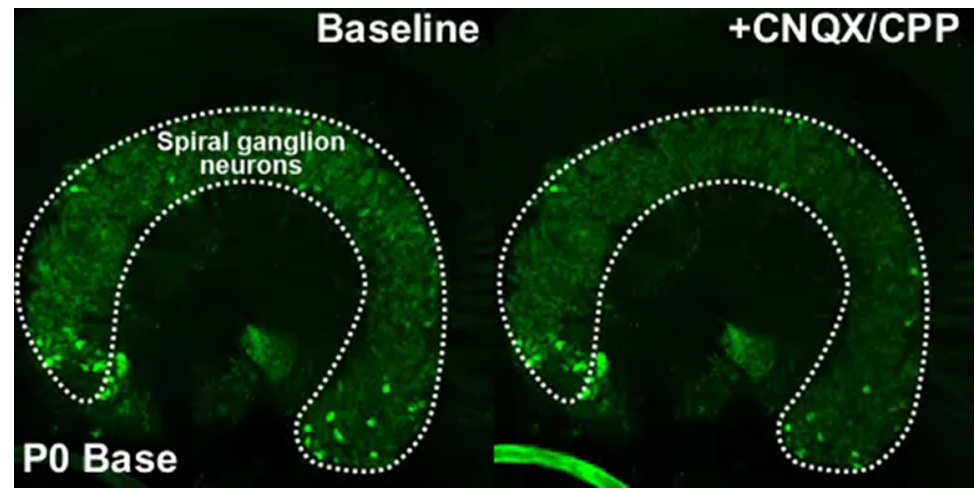

Movie 6. Correlated activation of SGNs requires IHC glutamate release. B, Time-lapse imaging of SGN activity in isolated basal sections of cochlea from PO Snap25-T2A-GCaMP6s mice. Application of CNQX/CPP (50/100 $\mu \mathrm{m})$ is indicated in the top right corner. [View online]

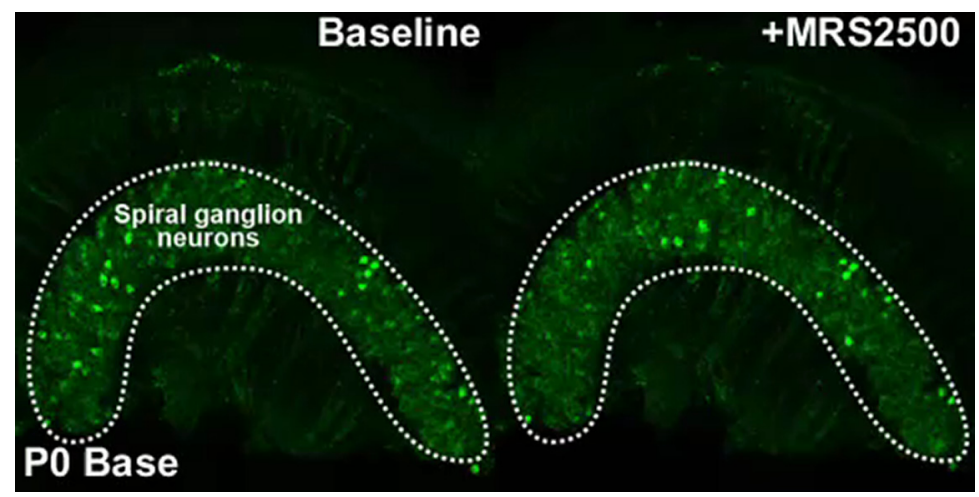

Movie 7. Correlated activation of SGNs requires P2ry1-mediated excitation of IHCS. A, Time-lapse imaging of SGN activity in isolated basal sections of cochlea from PO Snap25-T2A-GCaMP6s. Application of MRS2500 (1 $\mu \mathrm{M})$ is indicated in the top right corner. [View online]

processes, including SGN dendrites (Tritsch et al., 2007). Although synaptic excitation is required to induce burst firing of SGNs in WT mice, homeostatic increases in SGN membrane resistance, and thereby excitability in deaf mice (Vglut3 KOs), allows direct activation of groups of SGNs by these brief elevations of extracellular $\mathrm{K}^{+}$(Babola et al., 2018). Given that the membrane resistance of SGNs is extremely high at birth (Marrs and Spirou, 2012), it is possible that extruded $\mathrm{K}^{+}$could directly drive the activity of nearby SGNs at earlier developmental time points. To determine whether coordinated SGN Ca ${ }^{2+}$ transients require release of glutamate from IHCs, we applied the AMPAR and NMDAR antagonists CNQX and CPP to acutely isolated P0 preparations of basal cochleae (Fig. 8A,B; Movie 6). The number of coordinated events, the correlation coefficient between ROIs, the number of active ROIs, and the average frequency of transients per ROI were markedly decreased by CNQX/CPP (Fig. 8C), indicating that coordinated activation of SGNs at this early developmental stage also requires activation of ionotropic glutamate receptors. While coordinated SGN transients were abolished, individual SGNs exhibited infrequent $\mathrm{Ca}^{2+}$ transients when deprived of glutamatergic excitation, suggesting that there is a form of activity that is independent of synaptic excitation (Fig. $8 B$ ); however, this activity was not coordinated between neighboring SGNs, suggesting that it may arise through cell intrinsic processes.

Given the dependence of coordinated IHC activation on P2RY1-mediated ISC activity (Fig. 5B,C), coordinated SGN activity should also be sensitive to P2RY1 inhibition. Indeed, application of MRS2500 decreased the number of coordinated SGN transients, the correlation coefficient between ROIs, and the number of active ROIs (Fig. $8 D-F$; Movie 7). The average ROI transient frequency did not decrease in MRS2500, consistent with observations of increased, uncorrelated activity of IHCs with prolonged P2RY1 inhibition (Fig. 5E). Together, these data indicate that activation of P2RY1 on ISCs leads to IHC depolarization, glutamate release, and postsynaptic activation of SGNs when functional synapses first emerge at $\sim \mathrm{P} 0$.

\section{Developmental changes in spontaneous activity in the IC}

In the developing auditory midbrain (IC), bursts of activity originating in the cochlea coordinate the activity of neurons within isofrequency lamina (Babola et al., 2018), regions later responsive to specific frequencies of sounds. Events arising within one cochlea induce bilateral activity in both lobes of the IC, with the contralateral lobe exhibiting the strongest response, consistent with the known contralateral bias in information flow through the auditory pathway. While bursts of electrical activity have been detected as early as P1 in the auditory brainstem in anesthetized animals (Tritsch et al., 2010), little is known about how the spatial and temporal aspects of this activity change in vivo with development. To define developmental changes in IC activity, we performed time-lapse imaging of awake mice in Snap25-T2A-GCaMP6s mice. At all ages examined (from P1, the earliest age we could reliably perform imaging, to P16, just after hearing onset), periodic excitation of neurons occurred within isofrequency domains of both lobes of the IC (Fig. 9A,B; Movie 8). Bilateral events stochastically alternated between having larger amplitudes on the right and left, indicative of electrical activity coming from the left or right cochlea, respectively (Babola et al., 2018). The degree of lateralization (smaller/ larger amplitude) also varied on an event-by-event basis (degree of left/right dominance represented by dot sizes in Fig. 9C). On average, events were evenly balanced between left and right IC (Fig. 9C) and increased in frequency and amplitude with developmental age (Fig. 9D). To determine whether ambient room noise contributes to recorded neural activity after hearing onset $(\sim$ P12), two experiments were performed with the ear canals occluded (Fig. 9D, purple circles; P16). The frequency of $\mathrm{Ca}^{2+}$ transients in these animals decreased, indicating that soundevoked neural responses comprise a portion of the activity measured in animals without occlusion. The correlation between activity in the left and right lobes of the IC exhibited a small decrease at P10 (Fig. 9E). To determine the spatial extent of neuronal activation in IC, we calculated the average area activated during an IC event (defined by pixels that exhibited an amplitude response more than two-thirds of the maximum intensity; Fig. $9 F)$. The spatial spread of activity increased from P1 to P3, then slowly decreased over the next 2 postnatal weeks (Fig. 9E). These results indicate that auditory neurons within isofrequency domains experience a prolonged period of correlated activity before hearing onset and that the domains of active neurons 
A

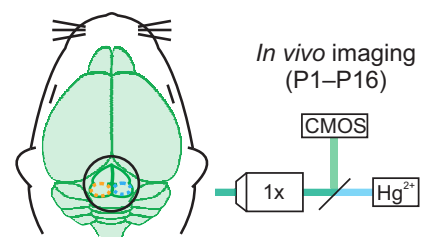

C

Snap25-2A-GCaMP6s

P1

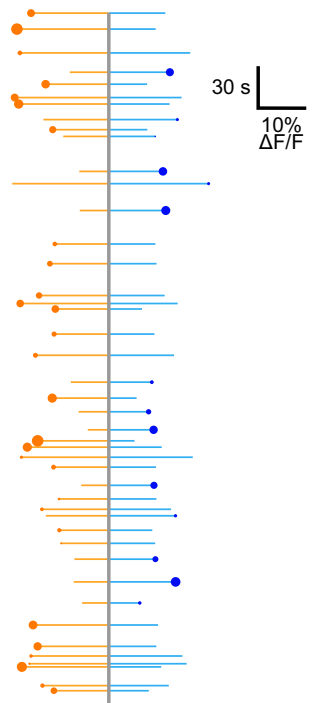

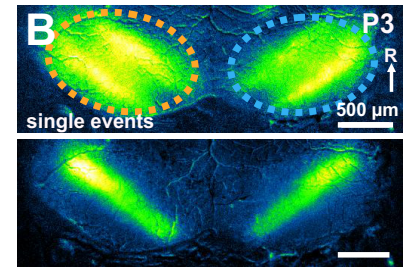

P7

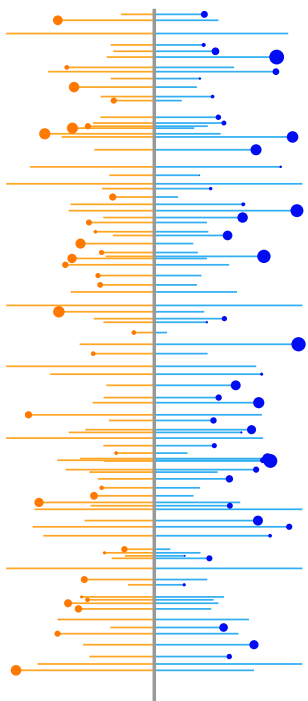

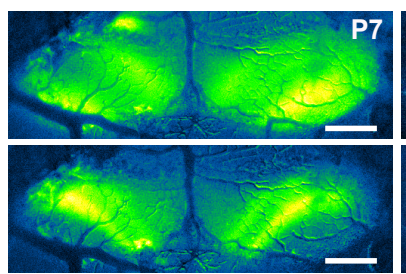

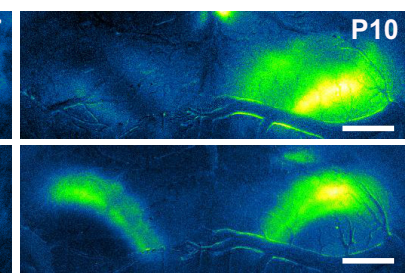

D
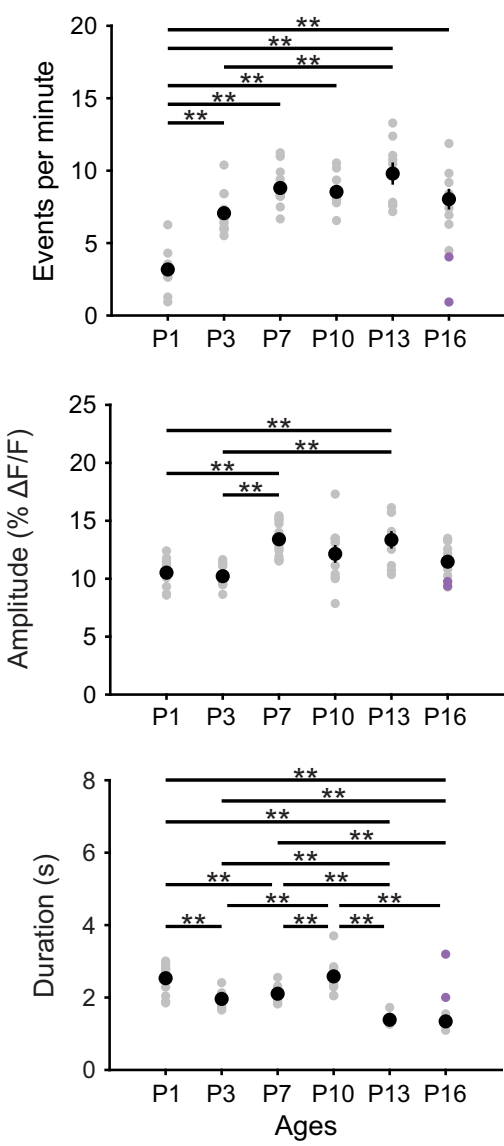

E

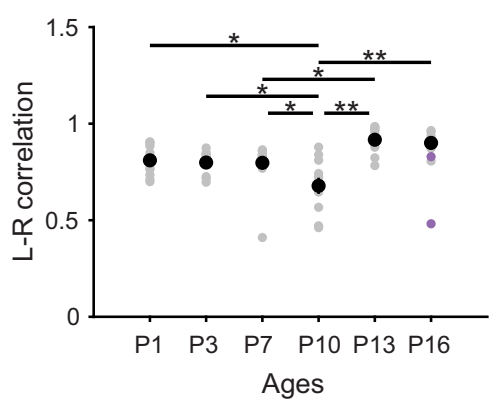

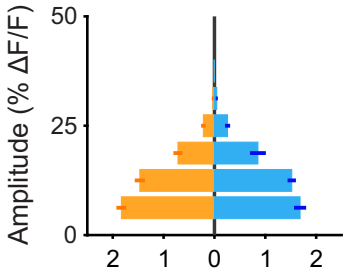

Dominant events per minute

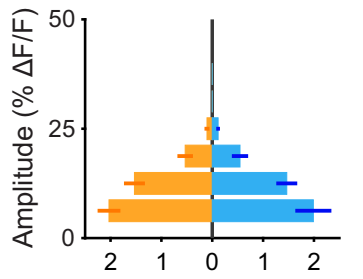

Dominant events per minute

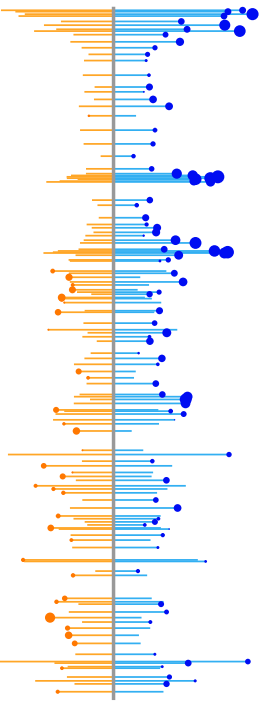

Ages

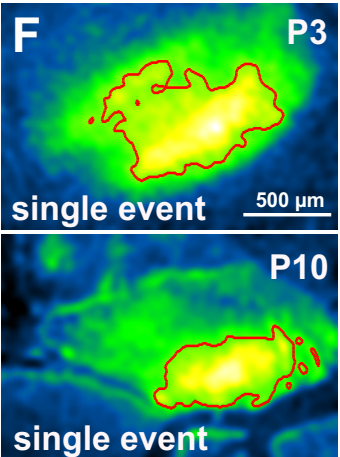

Figure 9. Developmental increase in spontaneous activity in the IC. A, Spontaneous neural activity monitored in unanesthetized mouse pups (Snap25-T2A-GCaMP6S) with wide-field epifluorescence. $\boldsymbol{B}$, Images of exemplar spontaneous $\mathrm{Ca}^{2+}$ transients in the auditory midbrain (IC) of unanesthetized Snap25-T2A-GCaMP6s mice (at P3, P7, and P10). Orange and blue ovals represent left and right IC, respectively, and correspond to ROIs used to examine fluorescence changes. Activity occurs within tonotopic bands (diagonal in each IC, rostral-lateral to caudal-medial), where single centrally located bands represent the lowest frequencies and lateral doublets represent progressively higher frequencies in mice after hearing onset (Babola et al., 2018). C, Graphs of activity over time for left (orange) and right (blue) lobes of the IC. Each line indicates an individual event. Circle represents which IC had the greater intensity. Size of dots indicates the difference in fluorescence between the two sides. Bottom, Histograms represent the number of dominant events per amplitude bin. $\boldsymbol{D}$, Quantification of frequency, amplitude, and duration (half-width of events) of events across different ages. Purple data points indicate experiments performed with middle ear occluded. $n=13 \mathrm{P} 1, n=12 \mathrm{P} 3, n=15 \mathrm{P} 7, n=11 \mathrm{P} 10, n=9 \mathrm{P} 13$, and $n=9 \mathrm{P} 16$ mice. $* * p<0.005$ (one-way ANOVA with Tukey post hoc). Comparisons not explicitly shown were not statistically significant. $E$, Quantification of the left and right IC correlation coefficient (Pearson) and average area of each event (calculated as the area of pixels with values $>2 / 3 \times$ max intensity) across different ages. Purple data points indicate experiments performed with middle ear occluded. $\boldsymbol{F}$, Example images of quantification of event areas $(2 / 3 \times$ max intensity delineated by red boundary) for single events. 


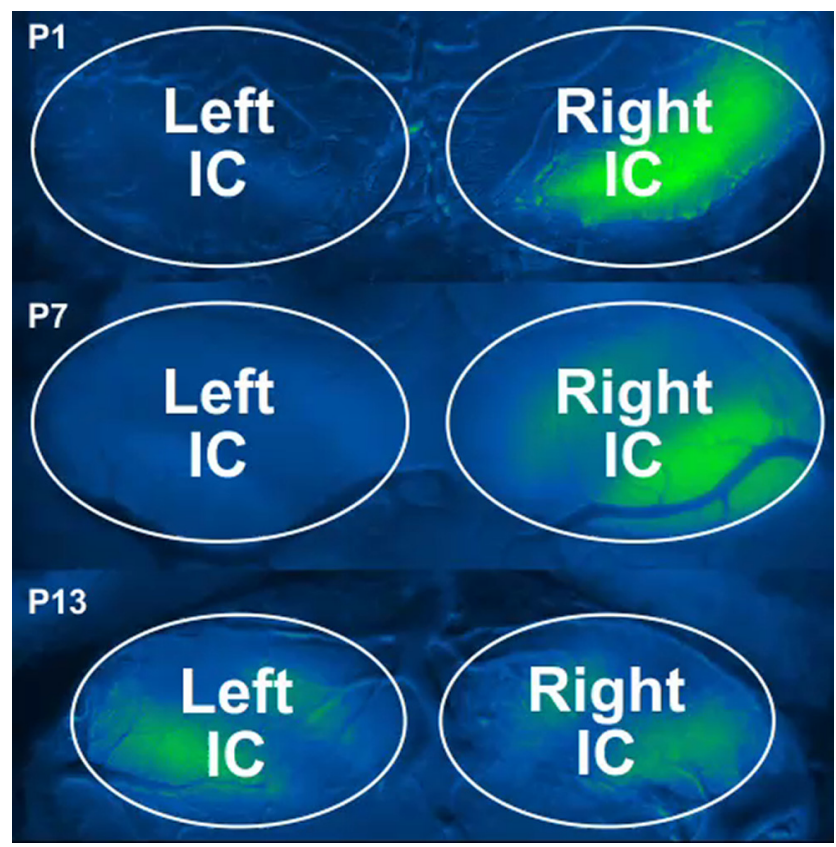

Movie 8. Developmental increase in spontaneous activity in the IC. $A$, Time-lapse imaging of IC activity in unanesthetized Snap25-T2A-GCaMP6s mice. [View online]

decrease with development, paralleling tonotopic refinement within the IC.

P2RY1 activity is required for spontaneous activity in vivoat $\mathrm{P} 1$ After the first postnatal week, spontaneous activity in the IC is sensitive to acute inhibition of P2RY1 (Babola et al., 2020). To determine whether P2RY1 is required for spontaneous activity in newborn animals, we performed time-lapse imaging of spontaneous activity in IC before and after acute injection MRS2500 (or vehicle) into the intraperitoneal space. Following MRS2500 injection, there was a significant decrease in the frequency ( $4.7 \pm 0.9$ events per minute in control, $2.49 \pm 0.5$ events per minute in MRS2500, Student's $t$ test with Bonferroni correction, $\left.t_{(6)}=4.07, p=0.01\right)$, but not the amplitude $(0.077 \pm 0.005 \mathrm{DF} / \mathrm{F}$ in control, $0.079 \pm 0.005 \mathrm{DF} / \mathrm{F}$ in MRS2500, Student's $t$ test with Bonferroni correction $t_{(6)}=0.33, p=0.76$ ), of spontaneous $\mathrm{Ca}^{2+}$ transients (Fig. 10D,F,G). In contrast, there were no significant changes in the frequency (Student's $t$ test with Bonferroni correction $t_{(7)}=0.52, p=0.62$ ) or amplitude (Student's $t$ test with Bonferroni correction $\left.t_{(7)}=2.07, p=0.08\right)$ after injection of vehicle alone (Fig. 10B,E,G).

Because injections were systemic and the permeability of MRS2500 across the blood-brain barrier is unknown, it is possible that a general suppression of neuronal activity could lead to the observed decrease in IC activity. However, some astrocytes in the CNS express P2RY1 receptors that, when activated, reduce local neuronal activity by enhancing $\mathrm{K}^{+}$uptake (F. Wang et al., 2012); thus, inhibition of P2RY1 would be expected to enhance, rather than inhibit, activity in the IC. Moreover, administration of MRS2500 did not alter the frequency or duration of retinal waveinduced activity in the superior colliculus (SC; Fig. 10G), suggesting that the effects observed in IC are because of selective manipulation of P2RY1 receptors in the auditory system. These in vivo results provide further evidence that P2Y1 autoreceptors within the cochlea initiate spontaneous bursts of neural activity in developing auditory centers from birth until the onset of hearing.

\section{Alpha 9-containing nAChRs modulate bilateral activity patterns in IC}

The results described above indicate that activation of P2RY1 on ISCs triggers a signaling cascade that coordinates the activity of nearby IHCs, SGNs, and central auditory neurons throughout the prehearing period. However, this is not the sole modulatory input to IHCs during this period. At this early stage of development, efferent cholinergic fibers form transient, inhibitory synapses on IHCs (Glowatzki and Fuchs, 2000), providing an additional means to shape IHC electrical activity. In ex vivo cochleae preparations, acute application of $\mathrm{nAChR}$ antagonists induces IHC burst firing, suggesting that release from cholinergic inhibition can initiate spontaneous bursts of activity (Johnson et al., 2011). Moreover, in vivo extracellular recordings from auditory brainstem neurons in anesthetized mice lacking the nAChRs in IHCs (Elgoyhen et al., 1994), exhibited bursts of action potentials at frequencies indistinguishable from controls, but bursts were shorter and contained more spikes (Clause et al., 2014), indicating that suppression of cholinergic inhibition of IHCs leads to altered burst firing of central auditory neurons. However, the influence of this efferent inhibitory input on the coordinated firing of auditory neurons in vivo in unanesthetized mice has not been examined. To explore the contribution of $\mathrm{nAChR} \alpha 9$ signaling to macroscopic patterns of activity in IC, we performed time-lapse imaging of spontaneous activity from both nAChR $\alpha 9 \mathrm{KO}\left(\alpha 9 \mathrm{KO}, \mathrm{Ch} r n \alpha 9^{-/-}\right)$and nAChR $\alpha 9$ gain-of-function ( $\alpha 9 \mathrm{GOF}$; Chrn $\alpha 9^{\mathrm{L} 9^{\mathrm{T}} \mathrm{T} / \mathrm{L} 9^{\prime} \mathrm{T}}$ or Chrn $\alpha 9^{\mathrm{L} 9^{9} \mathrm{~T} /+}$ ) mice (P7; Fig. $11 A-C$; Movie 9), which exhibit prolonged efferent currents with slower desensitization kinetics in IHCs (Taranda et al., 2009; Wedemeyer et al., 2018). The frequency of spontaneous events in IC was unchanged in both $\alpha 9 \mathrm{KO}$ and GOF mice relative to controls (one-way ANOVA, $F_{(3,45)}=0.46, p=0.71$; Fig. $11 F$ ). However, IC $\mathrm{Ca}^{2+}$ transients in homozygous $\alpha 9$ GOF mice were unexpectedly larger in amplitude than controls; $\alpha 9 \mathrm{KO}$ exhibited a trend toward lower amplitude $\mathrm{Ca}^{2+}$ transients, but this did not achieve significance (one-way ANOVA, $F_{(3,45)}=18.22, p=7 \mathrm{E}-8$; Tukey HSD, $p=0.06$; Fig. $11 F$ ). These changes are opposite of what would be predicted from simply relieving or enhancing the inhibitory effect of acetylcholine on IHCs (Glowatzki and Fuchs, 2000). Similarly, individual events in homozygous $\alpha 9$ GOF mice were longer (FWHM) than controls (one-way ANOVA, $F_{(3,45)}=3.2, p=0.032$; Tukey HSD, $p=0.026$; Fig. $\left.11 E, F\right)$, opposite of what would be predicted from greater inhibition of IHCs. There were also notable changes in the degree of lateralization among spontaneous IC events (Fig. 11C,D and Fig. 11F; $\mathrm{L}-\mathrm{R}$ correlation). Bilateral events were more symmetrical in $\alpha 9$ GOF and less symmetrical in $\alpha 9 \mathrm{KO}$ mice relative to controls (Fig. $11 F)$. Together, these results indicate that cholinergic efferent input to IHCs modulates the coordinated activity of central auditory neurons in unexpected ways to influence interhemispheric representation of cochlear activity before hearing onset.

\section{Discussion}

\section{Generation of coordinated neural activity by cochlear} supporting cells

Nascent neural networks exhibit highly stereotyped spontaneous activity, consisting of periods of high-frequency action potential firing interspersed with long periods of quiescence (Blankenship and Feller, 2010). Similar to the visual system, spontaneous activity generated in the cochlea begins just before birth in mice (Fig. 1), providing a prolonged period over which activity-dependent 
maturation and refinement can occur before hearing begins ( $\sim \mathrm{P} 12)$; however, much less is known about the mechanisms that initiate this spontaneous activity or how it changes over this developmental period. In contrast to the dynamic mechanisms responsible for retinal wave generation, our studies indicate that bursts in the auditory system are consistently driven by ISC purinergic signaling throughout development. Based on measures of spontaneous activity in vivo, each auditory neuron will experience $>30,000$ discrete bursts $\quad(\sim 2.0$ bursts/minute; $\sim 2900$ bursts/day) before hearing onset (Clause et al., 2014; Babola et al., 2018). Consistent with the stable generation of P2RY1-dependent bursts, neural activity in the IC remained highly stereotyped during this period, providing a means for activity-dependent, Hebbian plasticity.

\section{Purinergic signaling in the developing and adult cochlea}

Despite widespread expression of ionotropic P2X and metabotropic P2Y receptors in the developing cochlea (Nikolic et al., 2003; Lahne and Gale, 2008; Huang et al., 2010; Liu et al., 2015; Z. Wang et al., 2020), ISC electrical activity and structured burst firing of SGNs appears reliant primarily on P2RY1. The lack of P2X or other $\mathrm{G}_{\mathrm{q}^{-}}$ coupled $\mathrm{P} 2 \mathrm{Y}$ receptor activation may reflect the temporal and spatial characteristics of ATP release, which may occur in P2RY1-rich locations or yield ATP metabolites that favor P2RY1. However, burst firing persists in P2ry1 KO mice, in which IHCs are more depolarized (Babola et al., 2020), perhaps reflecting compensatory changes or activation of other purinergic receptors that normally elicit subthreshold responses.

A similar diversity of purinergic receptor expression is observed in the adult cochlea, including P2X, metabotropic P2Y, and adenosine P1 receptors (Housley et al., 2009; Huang et al., 2010). ATP receptor activation appears to play a neuroprotective role, as endolymphatic ATP increases following trauma and infusion of ATP into the inner ear profoundly reduces sound-evoked compound action potentials in the auditory nerve. While these effects may reflect shunting inhibition through P2X2 receptors (Housley et al., 2013), recent evidence indicates that supporting cells in the mature cochlea continue to exhibit large $\mathrm{Ca}^{2+}$ transients in response to exogenous ATP and UTP (Zhu and Zhao, 2010; Sirko et al., 2019). The role of this activity is unclear, but $\mathrm{Ca}^{2+}$ transients induced by mechanical damage and subsequent ATP release trigger ERK1/2 activation and promote IHC death in the developing cochlea (Lahne and Gale, 2008). If the developmental pathways described here reemerge following traumatic injury, purinergic receptor signaling could enhance $\mathrm{K}^{+}$

G
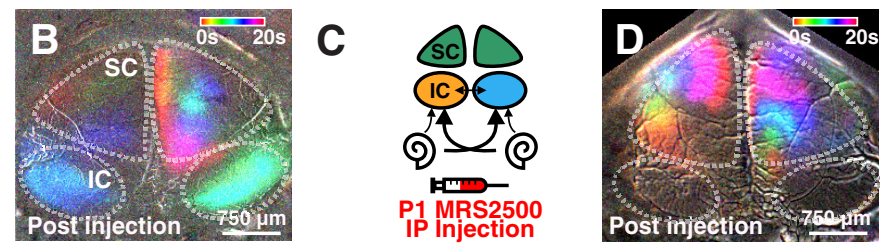

F
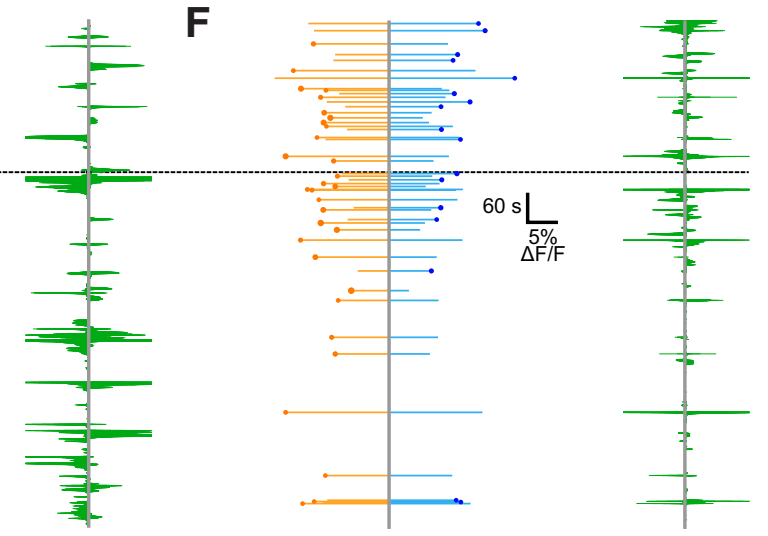
cochlea to midbrain. Sham solution (5\% mannitol) was injected via intraperitoneal catheter during imaging. IC: inferior colliculus, SC: superior colliculus, IP: intraperitoneal. $B, \mathrm{Ca}^{2+}$ transients in the IC and SC after injection of sham solution. Transients are colored based MPS2500 vocurrence. Segment after sham injection showing normal activity in both $I C$ and $S C$. $C$, Similar to $A$, but with injection of in left and right IC where each line indicates the fluorescence intensity of each detected event. Circle represents the dominant lobe. Size of the circle indicates the difference in fluorescence. Dashed line indicates time of injection. Right, $\mathrm{SC}$ activity before and after injection. Green shaded regions represent the number of active ROIs in the left and right SC. G, Quantification of IC event frequency and amplitude, and SC event frequency and duration. $n=8$ sham injected and $n=7$ MRS2500 injected Snap25-T2A-GCaMP6s mice. $* * p<0.05$; paired $t$ test with Bonferroni correction applied.

redistribution in the extracellular space, reduce IHC depolarization, and limit excitotoxic damage.

\section{The emergence of ATP-induced extracellular space changes in the cochlea}

ISC crenations dramatically increase the volume of extracellular space and speed $\mathrm{K}^{+}$redistribution, shaping the envelope of IHC excitation following P2RY1 activation (Babola et al., 2020). Their emergence may result from increasing levels of P2RY1 and TMEM16A as rapid increases in P2ryl promoter activity (Fig. 2), TMEM16A protein (H. C. Wang et al., 2015), and P2RY1 and TMEM16A mRNA (Scheffer et al., 2015; Kolla et al., 2020) occur over the first postnatal week. Accompanying these changes, the charge transfer of ISC spontaneous currents was similar across the first 2 postnatal weeks, despite decreasing event frequency (Fig. $1 D$ ), indicating a moderate increase in ion flux and enhanced osmotic force during each event over development. However, apical ISCs in P0 cochleae exhibited spontaneous currents as large as those observed at P7, and yet these ISCs did not crenate. 


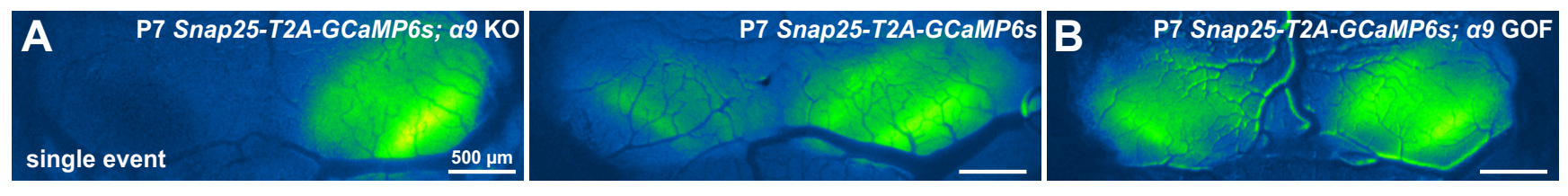

C
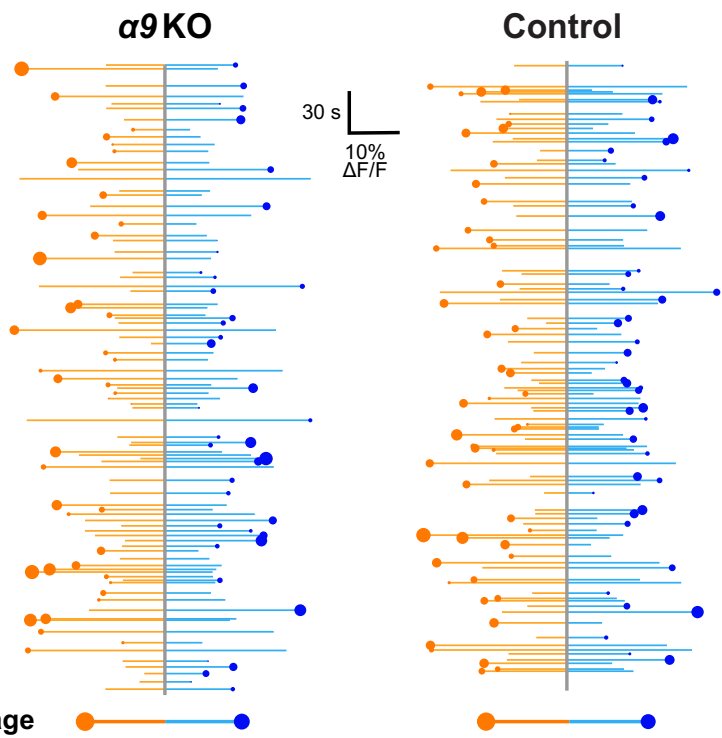

a9 GOF/+

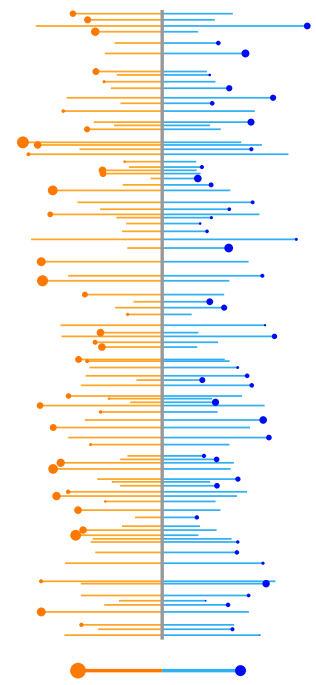

D
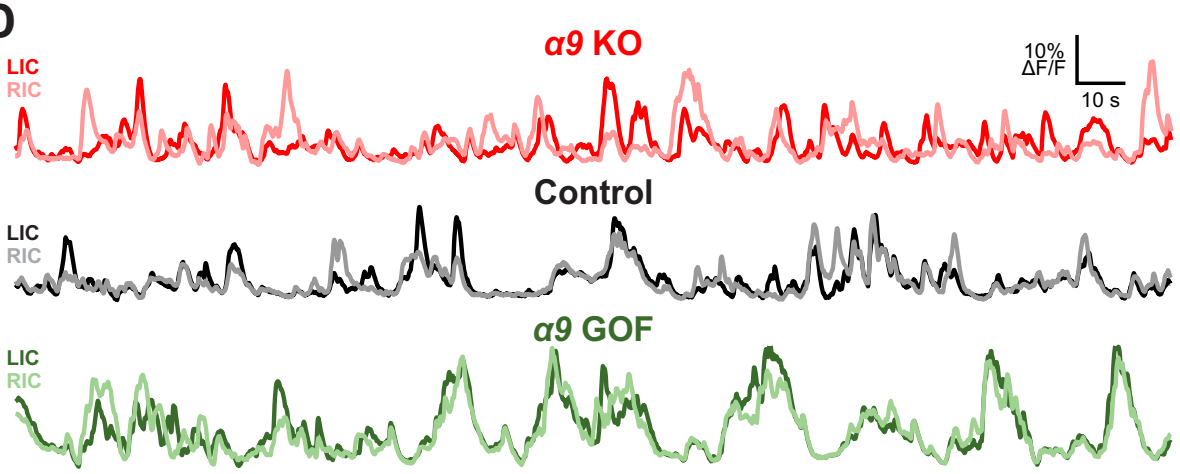

$\mathbf{E}$
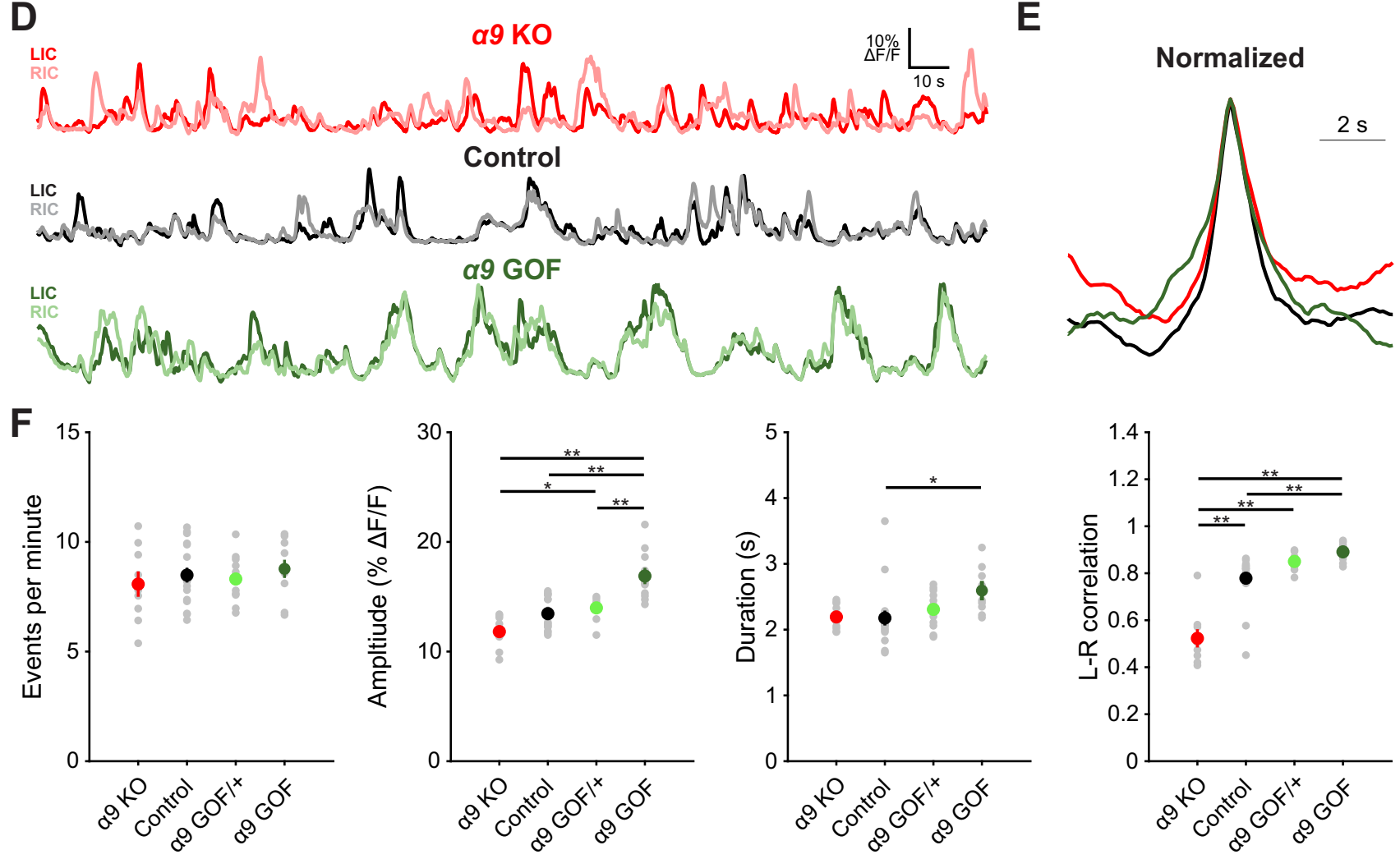

Figure 11. Cholinergic modulation of IHCs influences correlated activation of IC neurons before hearing onset. $A$, Exemplar spontaneous $\mathrm{Ca}^{2+}$ transient in the auditory midbrain (IC) of unanesthetized Snap25-T2A-GCaMP6s; Chrn $\alpha 9^{-/-}\left(\alpha 9\right.$ KO) and Snap25-T2A-GCaMP6s (control) mice at P7. B, Exemplar spontaneous $\mathrm{Ca}^{2+}$ transient in the auditory midbrain (IC) of unanesthetized Snap25-T2A-GCaMP6s; Chrn $\alpha 9^{\text {LTT/LTT }}(\alpha 9$ GOF) mice (P7). C, Graphs of activity over time for left (orange) and right (blue) lobes of the IC for indicated genotypes. Each line indicates an individual event. Circle represents which side had the greater intensity. Size of dots indicates the difference in fluorescence between the two sides. Bottom, Average event for each lobe of the IC. Size of circle is the average difference in the fluorescence between the two sides. $\boldsymbol{D}$, Example fluorescence traces for indicated genotypes. $\boldsymbol{E}$, Average event from traces shown in $\boldsymbol{D}$ normalized to amplitude. $\boldsymbol{F}$, Quantification of event frequency, amplitude, duration, and left-right correlation coefficient (Pearson) across indicated genotypes. $n=9 \alpha 9 \mathrm{~K} 0, n=17$ control, $n=13 \alpha 9 \mathrm{GOF} /+$, and $n=10 \alpha 9 \mathrm{GOF}$ mice. $* * p<0.005 ; * p<0.05$; one-way ANOVA with Tukey post hoc. Comparisons not shown are not statistically significant. 


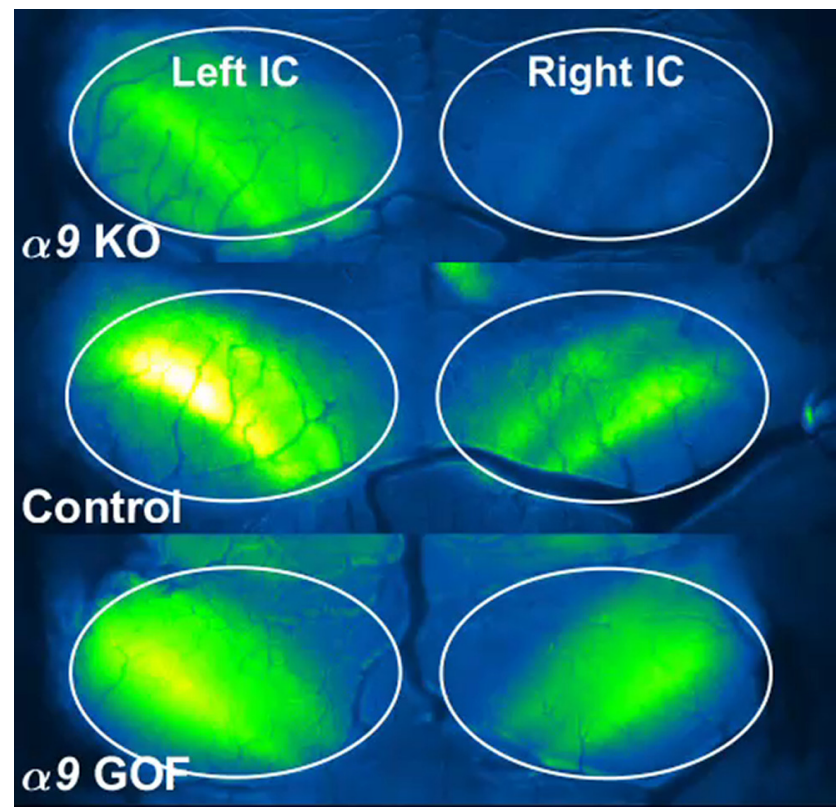

Movie 9. Cholinergic modulation of IHCs influences correlated activation of IC neurons before hearing onset. $\boldsymbol{A}$, Time-lapse imaging of IC activity in unanesthetized, P7 Snap25-T2AGCaMP6s; Chrn $\alpha 9^{-/-}(\alpha 9 \mathrm{KO})$ and Chrn $\alpha 9^{\text {L9T/L9T }}(\alpha 9 \mathrm{GOF})$ mice. [View online]

While changes in ion flux may contribute to the emergence of crenations, expression of aquaporins, a family of highly permeable water channels that enable rapid diffusion of water across biological membranes (Reuss, 2012), may also regulate this process. Recent single-cell RNAseq analysis of the cochlear epithelium revealed that AQP4 and AQP11 genes are expressed within Kölliker's organ after the first postnatal week (Kolla et al., 2020). AQP11 is a nontraditional aquaporin family member that is localized to the ER membrane (Morishita et al., 2005), has a higher permeability to glycerol than water (Madeira et al., 2014), and is expressed at relatively stable levels throughout cochlear development (Kolla et al., 2020), making it an unlikely candidate to regulate water movement. AQP4 is highly permeable to water and its expression dramatically increases between P1 and P7 (Kolla et al., 2020), indicating that AQP4 could enable the large water movements that underlie ISC crenation and may similarly be expressed in a base to apex progression.

Recent evidence suggests that ISC control of the extracellular space influences the activation of IHCs during spontaneous events and controls IHC excitability. Both conditional removal of TMEM16A from the cochlea (H. C. Wang et al., 2015) or acute inhibition of P2RY1 (Babola et al., 2020) prevents ISC crenation during spontaneous events. The subsequent collapse of the extracellular space limits $\mathrm{K}^{+}$diffusion, reducing the number of IHCs activated per ISC $\mathrm{Ca}^{2+}$ transient and promoting local $\mathrm{K}^{+}$buildup that eventually leads to tonic IHC firing. Therefore, the lack of crenation at earlier stages may slow $\mathrm{K}^{+}$redistribution to induce larger and more prolonged depolarization of IHCs at a time when ribbon synapses are immature and $\mathrm{Ca}^{2+}$ channel expression is low (Marcotti, 2012; Michanski et al., 2019).

\section{Involvement of cholinergic efferents in modulating early spontaneous activity}

In the developing cochlea, IHCs are transiently innervated by efferent fibers, which provide powerful inhibitory input (Glowatzki and Fuchs, 2000). Previous studies in isolated cochleae demonstrated that temporarily relieving this inhibition produced bursts of action potentials in IHCs (Johnson et al., 2011), suggesting that transient efferent activity could alone initiate burst firing. However, auditory brainstem neurons in (anesthetized) $\alpha 9 \mathrm{KO}$ mice exhibit prominent burst firing in vivo, with bursts occurring at similar frequencies, but with shorter durations and containing more action potentials than controls (Clause et al., 2014). Moreover, $\alpha 9$ GOF mice that have enhanced efferent inhibition of IHCs (Wedemeyer et al., 2018) also exhibit spontaneous action potentials in the auditory brainstem, although at lower frequencies than controls (Di Guilmi et al., 2019). Our in vivo macroscopic imaging studies indicate that periodic excitation of auditory midbrain neurons occurred in $\alpha 9 \mathrm{KO}$ mice at the same frequency as controls (Fig. 11), providing additional evidence that efferent input is not required to initiate burst firing (Clause et al., 2014). However, manipulating $\alpha 9 \mathrm{nAChR}$ signaling altered the lateralization (contralateral bias) of activity in the auditory midbrain. In $\alpha 9$ $\mathrm{KO}$ mice, bilateral activation of the IC was more asymmetric than controls (Fig. 11A,F); and, conversely, activity in $\alpha 9 \mathrm{GOF}$ mice was more symmetric (Fig. $11 B, F$ ). Changes in propagation of activity to both hemispheres may result from efferent-mediated alterations in precise burst firing patterns (Clause et al., 2014) but could also reflect changes in the developmental profile of cochlear cells (Turcan et al., 2010), developmental alterations in the electrophysiological properties of auditory neurons (Di Guilmi et al., 2019), or refinement deficits that arise through altered activity in these mice (Zhang et al., 2011; Clause et al., 2014). Surprisingly, the effects on IC neuronal burst firing in $\alpha 9 \mathrm{KO}$ and $\alpha 9 \mathrm{GOF}$ mice were opposite of that predicted based on the inhibitory effect of acetylcholine on IHCs (Glowatzki and Fuchs, 2000), with enhanced inhibition of IHCs $\alpha 9$ GOF mice resulting in prolonged, larger-amplitude events in central auditory neurons (Fig. 11). Activity patterns in these mice could reflect compensatory shifts in excitation along the auditory pathway, similar to changes in excitability observed in Vglut3 KO mice (Babola et al., 2018). Together, these results provide additional evidence that the efferent system is not required to initiate spontaneous burst firing in the developing auditory system, but rather plays an active role in shaping early sensory-independent activity, raising the possibility that specific patterns of activity are required to induce proper maturation of sound-processing circuits.

\section{The role of spontaneous neural activity in development}

Barrel fields fail to form when thalamic input to the somatosensory cortex is silenced during early postnatal life (AntónBolaños et al., 2019), and genetic disruption of retinal waves leads to profuse retinal ganglion cell axon arborization in SC and segregation deficits in the thalamus (Rossi et al., 2001), demonstrating the critical role of early patterned activity in circuit maturation. In the auditory system, similar refinement deficits have been observed in $\alpha 9 \mathrm{KO}$ mice and in various models of deafness. However, the functional consequences of these manipulations on sensory performance remain underexplored. $\alpha 9 \mathrm{KO}$ mice exhibit deficits in sound localization tasks (Clause et al., 2017), but whether these changes are because of disruption in spontaneous activity or the lack of a functional efferent system after hearing onset remains uncertain. Insight into the mechanisms that govern spontaneous activity in the auditory system provides an experimental framework for selectively disrupting early spontaneous activity, while preserving hearing, 
allowing assessment of the role of stereotyped burst firing in development.

\section{References}

Antón-Bolaños N, Sempere-Ferràndez A, Guillamón-Vivancos T, Martini FJ, Pérez-Saiz L, Gezelius H, Filipchuk A, Valdeolmillos M, López-Bendito G (2019) Prenatal activity from thalamic neurons governs the emergence of functional cortical maps in mice. Science 364:987-990.

Babola TA, Kersbergen CJ, Wang HC, Bergles DE (2020) Purinergic signaling in cochlear supporting cells reduces hair cell excitability by increasing the extracellular space. Elife 9:e52160.

Babola TA, Li S, Gribizis A, Lee BJ, Issa JB, Wang HC, Crair MC, Bergles DE (2018) Homeostatic control of spontaneous activity in the developing auditory system. Neuron 99:511-524.

Blankenship AG, Feller MB (2010) Mechanisms underlying spontaneous patterned activity in developing neural circuits. Nat Rev Neurosci 11:18-29.

Brandt A, Striessnig J, Moser T (2003) CaV1.3 channels are essential for development and presynaptic activity of cochlear inner hair cells. J Neurosci 23:10832-10840.

Chen P, Johnson JE, Zoghbi HY, Segil N (2002) The role of Math1 in inner ear development: uncoupling the establishment of the sensory primordium from hair cell fate determination. Development 129:24952505.

Clause A, Kim G, Sonntag M, Weisz CJ, Vetter DE, Rübsamen R, Kandler K (2014) The precise temporal pattern of prehearing spontaneous activity is necessary for tonotopic map refinement. Neuron 82:822835.

Clause A, Lauer AM, Kandler K, Morley BJ (2017) Mice lacking the alpha9 subunit of the nicotinic acetylcholine receptor exhibit deficits in frequency difference limens and sound localization. Front Cell Neurosci 11:167.

Di Guilmi MN, Boero LE, Castagna VC, Rodríguez-Contreras A, Wedemeyer C, Gómez-Casati ME, Elgoyhen AB (2019) Strengthening of the efferent olivocochlear system leads to synaptic dysfunction and tonotopy disruption of a central auditory nucleus. J Neurosci 39:7037-7048.

Driver EC, Kelley MW (2010) Transfection of mouse cochlear explants by electroporation. Curr Protoc Neurosci 4.34:1-10.

Elgoyhen AB, Johnson DS, Boulter J, Vetter DE, Heinemann S (1994) $\alpha 9$ : an acetylcholine receptor with novel pharmacological properties expressed in rat cochlear hair cells. Cell 79:705-715.

Feller MB, Wellis DP, Stellwagen D, Werblin FS, Shatz CJ (1996) Requirement for cholinergic synaptic transmission in the propagation of spontaneous retinal waves. Science 272:1182-1187.

Firth SI, Wang CT, Feller MB (2005) Retinal waves: mechanisms and function in visual system development. Cell Calcium 37:425-432.

Glowatzki E, Fuchs PA (2000) Cholinergic synaptic inhibition of inner hair cells in the neonatal mammalian cochlea. Science 288:23662368.

Gribizis A, Ge X, Daigle TL, Ackman JB, Zeng H, Lee D, Crair MC (2019) Visual cortex gains independence from peripheral drive before eye opening. Neuron 104:711-723.e3.

Hinojosa R (1977) A note on development of Corti's organ. Acta Otolaryngol 84:238-251.

Housley GD, Bringmann A, Reichenbach A (2009) Purinergic signaling in special senses. Trends Neurosci 32:128-141.

Housley GD, Morton-Jones R, Vlajkovic SM, Telang RS, Paramananthasivam V, Tadros SF, Wong AC, Froud KE, Cederholm JM, Sivakumaran Y, Snguanwongchai P, Khakh BS, Cockayne DA, Thorne PR, Ryan AF (2013) ATP-gated ion channels mediate adaptation to elevated sound levels. Proc Natl Acad Sci USA 110:7494-7499.

Houston D, Ohno M, Nicholas RA, Jacobson KA, Harden TK (2006) 2-iodoN6-methyl-(N)-methanocarba-2'-deoxyadenosine-3', $\quad 5^{\prime}$-bisphosphate ([32P] MRS2500), a novel radioligand for quantification of native P2Y1 receptors. Br J Pharmacol 147:459-467.

Huang LC, Thorne PR, Vlajkovic SM, Housley GD (2010) Differential expression of P2Y receptors in the rat cochlea during development. Purinergic Signal 6:231-248.

Jagger DJ, Forge A (2006) Compartmentalized and signal-selective gap junctional coupling in the hearing cochlea. J Neurosci 26:1260-1268.
Johnson SL, Eckrich T, Kuhn S, Zampini V, Franz C, Ranatunga KM, Roberts TP, Masetto S, Knipper M, Kros CJ, Marcotti W (2011) Positiondependent patterning of spontaneous action potentials in immature cochlear inner hair cells. Nat Neurosci 14:711-717.

Johnson SL, Kennedy HJ, Holley MC, Fettiplace R, Marcotti W (2012) The resting transducer current drives spontaneous activity in prehearing mammalian cochlear inner hair cells. J Neurosci 32:1047910483.

Kamiya K, Yum SW, Kurebayashi N, Muraki M, Ogawa K, Karasawa K, Miwa A, Guo X, Gotoh S, Sugitani Y, Yamanaka H, Ito-Kawashima S, Iizuka T, Sakurai T, Noda T, Minowa O, Ikeda K (2014) Assembly of the cochlear gap junction macromolecular complex requires connexin 26. J Clin Invest 124:1598-1607.

Kolla L, Kelly MC, Mann ZF, Anaya-Rocha A, Ellis K, Lemons A, Palermo AT, So KS, Mays J, Orvis J, Burns JC, Hertzano R, Driver EC, Kelley MW (2020) Characterization of the development of the cochlear epithelium at the single cell level. Nat Commun 11:2389.

Lahne M, Gale JE (2008) Damage-induced activation of ERK1/2 in cochlear supporting cells is a hair cell death-promoting signal that depends on extracellular ATP and calcium. J Neurosci 28:4918-4928.

Lippe W (1994) Rhythmic spontaneous activity in the developing avian auditory system. J Neurosci 14:1486-1495.

Liu C, Glowatzki E, Fuchs PA (2015) Unmyelinated type II afferent neurons report cochlear damage. Proc Natl Acad Sci USA 112:14723-14727.

Madeira A, Fernández-Veledo S, Camps M, Zorzano A, Moura TF, Ceperuelo-Mallafré V, Vendrell J, Soveral G (2014) Human aquaporin-11 is a water and glycerol channel and localizes in the vicinity of lipid droplets in human adipocytes. Obesity (Silver Spring) 22:2010-2017.

Marcotti W (2012) Functional assembly of mammalian cochlear hair cells. Exp Physiol 97:438-451.

Marrs GS, Spirou GA (2012) Embryonic assembly of auditory circuits: spiral ganglion and brainstem. J Physiol 590:2391-2408.

Michanski S, Smaluch K, Steyer AM, Chakrabarti R, Setz C, Oestreicher D, Fischer C, Möbius W, Moser T, Vogl C, Wichmann C (2019) Mapping developmental maturation of inner hair cell ribbon synapses in the apical mouse cochlea. Proc Natl Acad Sci USA 116:6415-6424.

Morishita Y, Matsuzaki T, Hara-chikuma M, Andoo A, Shimono M, Matsuki A, Kobayashi K, Ikeda M, Yamamoto T, Verkman A, Kusano E, Ookawara S, Takata K, Sasaki S, Ishibashi K (2005) Disruption of aquaporin-11 produces polycystic kidneys following vacuolization of the proximal tubule. Mol Cell Biol 25:7770-7779.

Nikolic P, Housley GD, Thorne PR (2003) Expression of the P2X7 receptor subunit of the adenosine 5'-triphosphate-gated ion channel in the developing and adult rat cochlea. Audiol Neurootol 8:28-37.

Reuss L (2012) Water Transport Across Cell Membranes. In eLS, (Ed.). https://doi.org/10.1002/9780470015902.a0020621.pub2.

Rossi FM, Pizzorusso T, Porciatti V, Marubio LM, Maffei L, Changeux JP (2001) Requirement of the nicotinic acetylcholine receptor $\beta 2$ subunit for the anatomical and functional development of the visual system. Proc Natl Acad Sci USA 98:6453-6458.

Scheffer DI, Shen J, Corey DP, Chen ZY (2015) Gene expression by mouse inner ear hair cells during development. J Neurosci 35:63666380

Seal RP, Akil O, Yi E, Weber CM, Grant L, Yoo J, Clause A, Kandler K, Noebels JL, Glowatzki E, Lustig LR, Edwards RH (2008) Sensorineural deafness and seizures in mice lacking vesicular glutamate transporter 3 . Neuron 57:263-275.

Sirko P, Gale JE, Ashmore JF (2019) Intercellular $\mathrm{Ca}^{2+}$ signalling in the adult mouse cochlea. J Physiol 597:303-317.

Sonntag M, Englitz B, Kopp-Scheinpflug C, Rübsamen R (2009) Early postnatal development of spontaneous and acoustically evoked discharge activity of principal cells of the medial nucleus of the trapezoid body: an in vivo study in mice. J Neurosci 29:9510-9520.

Sun S, Babola T, Pregernig G, So KS, Nguyen M, Su SM, Palermo AT, Bergles DE, Burns JC, Müller U (2018) Hair cell mechanotransduction regulates spontaneous activity and spiral ganglion subtype specification in the auditory system. Cell 174:1247-1263.e15.

Taranda J, Maison SF, Ballestero JA, Katz E, Savino J, Vetter DE, Boulter J, Liberman MC, Fuchs PA, Elgoyhen AB (2009) A point mutation in the hair cell nicotinic cholinergic receptor prolongs cochlear inhibition and enhances noise protection. PLoS Biol 7:e1000018. 
Tiriac A, Smith BE, Feller MB (2018) Light prior to eye opening promotes retinal waves and eye-specific segregation. Neuron 100:10591065.e4.

Tritsch NX, Bergles DE (2010) Developmental regulation of spontaneous activity in the mammalian cochlea. J Neurosci 30:1539-1550.

Tritsch NX, Yi E, Gale JE, Glowatzki E, Bergles DE (2007) The origin of spontaneous activity in the developing auditory system. Nature 450:50-55.

Tritsch NX, Rodríguez-Contreras A, Crins TH, Wang HC, Borst JG, Bergles DE (2010) Calcium action potentials in hair cells pattern auditory neuron activity before hearing onset. Nat Neurosci 13:1050-1052.

Turcan S, Slonim DK, Vetter DE (2010) Lack of nAChR activity depresses cochlear maturation and up-regulates GABA system components: temporal profiling of gene expression in $\alpha 9$ null mice. PLoS One 5:e9058.

Wang F, Smith NA, Xu Q, Fujita T, Baba A, Matsuda T, Takano T, Bekar L, Nedergaard M (2012) Astrocytes modulate neural network activity by $\mathrm{Ca}^{2+}$-dependent uptake of extracellular $\mathrm{K}^{+}$. Sci Signal 5:ra26.

Wang HC, Lin CC, Cheung R, Zhang-Hooks Y, Agarwal A, Ellis-Davies G, Rock J, Bergles DE (2015) Spontaneous activity of cochlear hair cells triggered by fluid secretion mechanism in adjacent support cells. Cell 163:1348-1359.

Wang Z, Jung JS, Inbar TC, Rangoussis KM, Faaborg-Andersen C, Coate TM (2020) The purinergic receptor P2rx3 is required for spiral ganglion neuron branch refinement during development. eNeuro 7:ENEURO.017920.2020
Wedemeyer C, Vattino LG, Moglie MJ, Ballestero J, Maison SF, Di Guilmi MN, Taranda J, Liberman MC, Fuchs PA, Katz E, Elgoyhen AB (2018) A gain-of-function mutation in the $\alpha 9$ nicotinic acetylcholine receptor alters medial olivocochlear efferent short-term synaptic plasticity. J Neurosci 38:3939-3954

Xu HP, Furman M, Mineur YS, Chen H, King SL, Zenisek D, Zhou ZJ, Butts DA, Tian N, Picciotto MR, Crair MC (2011) An instructive role for patterned spontaneous retinal activity in mouse visual map development. Neuron 70:1115-1127.

Zampini V, Johnson SL, Franz C, Knipper M, Holley MC, Magistretti J, Russo G, Marcotti W, Masetto S (2014) Fine tuning of CaV1.3 $\mathrm{Ca}^{2+}$ channel properties in adult inner hair cells positioned in the most sensitive region of the gerbil cochlea. PLoS One 9: e113750.

Zhang-Hooks YX, Agarwal A, Mishina M, Bergles DE (2016) NMDA receptors enhance spontaneous activity and promote neuronal survival in the developing cochlea. Neuron 89:337-350.

Zhang J, Ackman JB, Xu HP, Crair MC (2011) Visual map development depends on the temporal pattern of binocular activity in mice. Nat Neurosci 15:298-307.

Zheng QY, Johnson KR, Erway LC (1999) Assessment of hearing in 80 inbred strains of mice by ABR threshold analyses. Hear Res 130:94-107.

Zhu Y, Zhao HB (2010) ATP-mediated potassium recycling in the cochlear supporting cells. Purinergic Signal 6:221-229. 